\title{
Local strong solutions to a quasilinear degenerate fourth-order thin-film equation
}

\author{
Christina Lienstromberg® and Stefan Müller
}

\begin{abstract}
We study the problem of existence and uniqueness of strong solutions to a degenerate quasilinear parabolic non-Newtonian thin-film equation. Originating from a non-Newtonian Navier-Stokes system, the equation is derived by lubrication theory and under the assumption that capillarity is the only driving force. The fluid's shear-thinning rheology is described by the so-called Ellis constitutive law. For flow behaviour exponents $\alpha \geq 2$ the corresponding initial boundary value problem fits into the abstract setting of Amann (Function Spaces, Differential Operators and Nonlinear Analysis, Vieweg Teubner Verlag, Stuttgart, 1993). Due to a lack of regularity this is not true for flow behaviour exponents $\alpha \in(1,2)$. For this reason we prove an existence theorem for abstract quasilinear parabolic evolution problems with Hölder continuous dependence. This result provides existence of strong solutions to the non-Newtonian thinfilm problem in the setting of fractional Sobolev spaces and (little) Hölder spaces. Uniqueness of strong solutions is derived by energy methods and by using the particular structure of the equation.
\end{abstract}

Mathematics Subject Classification. 35K25, 35K35, 35K55, 35K59, 35K65, 35Q35, 76A05, 76A20, 76D03.

Keywords. Thin-film equation, Non-Newtonian fluid, Classical solution, Quasilinear parabolic equation.

\section{Introduction}

This contribution is motivated by questions for existence and uniqueness of strong solutions to the degenerate quasilinear fourth-order evolution problem

$$
\begin{cases}u_{t}+a\left(u^{3}\left[1+\left|b u u_{x x x}\right|^{\alpha-1}\right] u_{x x x}\right)_{x}=0, & t>0, x \in \Omega:=(-l, l) \\ u_{x}=u_{x x x}=0, & t>0, x \in \partial \Omega \\ u(0, \cdot)=u_{0}(\cdot), & x \in \Omega,\end{cases}
$$


describing the evolution of the height $u(t, x)$ of a non-Newtonian incompressible thin liquid film on a solid bottom. Here $a, b>0$ denote positive constants that depend on the fluid's properties and are specified later. The exponent $\alpha$ describes the fluid's flow behaviour. Problem (1.1) is derived by applying lubrication theory $[20,34]$ to the non-Newtonian Navier-Stokes system. We use the so-called Ellis constitutive law $[32,43]$ to describe the fluid's shear-thinning rheology. Observe that the fluid is Newtonian if $\alpha=1$. In this case we recover from $(1.1)_{1}$ the well-known thin-film equation

$$
u_{t}+a\left(u^{3} u_{x x x}\right)_{x}=0, \quad t>0, x \in \Omega .
$$

In this paper we treat the case $\alpha>1$, in which the fluid is said to be shearthinning. Moreover, we assume the fluid's dynamics to be driven by capillary forces only. In particular gravitational forces are neglected; c.f. Sect. 2 for a more detailed review of the derivation of the governing equations.

There is a rich literature on weak solutions of equations related to (1.1). The first pioneering results on the classical Newtonian thin-film equation

$$
u_{t}+a\left(u^{n} u_{x x x}\right)_{x}=0, \quad n \in \mathbb{N},
$$

go back to BERNIS and FRIEDMANN [8]. There the authors prove in particular the existence of global non-negative weak solutions, as well as positivity and uniqueness for $n \geq 4$. Among others, also the works [9-11,15] can be mentioned in the context of global non-negative weak solutions. For contributions to the study of non-Newtonian fluids the reader shall be in particular referred to the works $[25,26]$ of KING, where non-Newtonian generalisations of (1.2) are investigated. In particular the author studies the doubly nonlinear equation

$$
u_{t}+\left(u^{n}\left|u_{x x x}\right|^{p-2} u_{x x x}\right)_{x}=0,
$$

describing for $p \neq 2$ the spreading of so-called power-law or Ostwald-de Waele fluids. In the work [6] ANsini and GiACOMELLI establish the existence of global non-negative weak solutions to (1.3) for $p>2$ and $\frac{p-1}{2}<n<2 p-1$. In [5] the same authors verify the existence of travelling-wave solutions and study a class of quasi-self-similar solutions to (1.1). Moreover, in [33] the authors establish the existence of weak solutions to a non-Newtonian Stokes equation with a viscosity that depends on the fluid's shear rate and its pressure at the same time.

Uniqueness of weak solutions to the Newtonian thin-film equation (1.2) for $n<4$ is still an open problem. Moreover, due to their weak regularity properties, weak solutions do usually not provide much control at the contact points, where the film height $u$ tends to zero, i.e. where solid, liquid (and gas) meet . These are probably the reasons why existence and uniqueness of non-negative strong solutions of the free-boundary value problem associated to (1.2) have recently attracted much attention. The free boundary is the boundary $\partial\{u>0\}$ of the solution's support. The main difficulty in the analysis is again caused by the fact that the equation's parabolicity degenerates, as the film height $u$ tends to zero. A rich amount of research has been dedicated to this problem in the last decade, mostly prescribing a constant slope at the free 
boundary; see [13,16-19,22-24,27-29,36] for strong solutions in (weighted) Sobolev or Hölder spaces.

In this paper we focus onpositive strong solutions, emanating from a positive initial film height. To construct such solutions it is convenient to give up the divergence form of (1.1), in order to emphasise the quasilinear structure. For sufficiently regular solutions, (1.1) is equivalent to

$$
u_{t}+A\left(u, u_{x}, u_{x x}, u_{x x x}\right) u_{x x x x}=F\left(u, u_{x}, u_{x x}, u_{x x x}\right), \quad t>0, x \in \Omega,
$$

where

$$
\begin{aligned}
& A\left(u, u_{x}, u_{x x}, u_{x x x}\right)=a u^{3}\left(1+\alpha\left|b u u_{x x x}\right|^{\alpha-1}\right) \text { and } \\
& F\left(u, u_{x}, u_{x x}, u_{x x x}\right)=-3 a u^{2}\left(1+\left|\tilde{b} u u_{x x x}\right|^{\alpha-1}\right) u_{x} u_{x x x} .
\end{aligned}
$$

In (1.4) the highest-order term $u_{x x x x}$ appears only linearly, while the nonlinearity $\left|u_{x x x}\right|^{\alpha-1} u_{x x x}$ is on the right-hand side. However, the coefficient function $A$ contains the delicate nonlinearity $\left|u_{x x x}\right|^{\alpha-1}$.

Regarding the existence and uniqueness of strong solutions to (1.1), it turns out that there is a qualitative difference between flow behaviour exponents $\alpha \in(1,2)$ and those larger than or equal 2. If we associate to (1.4) an abstract quasilinear Cauchy problem

$$
\left\{\begin{array}{l}
\dot{u}+\mathcal{A}(u) u=\mathcal{F}(u), \quad t>0 \\
u(0)=0,
\end{array}\right.
$$

it turns out that in the latter case $\alpha \geq 2$ the operator $\mathcal{A}$ and the right-hand side $\mathcal{F}$ are Lipschitz continuous in an appropriate sense and the classical Hölder theory of EIDEL'MAN [14, Thm. III.4.6.3] as well as the abstract theory of Amann [4, Thm. 12.1] are applicable and provide existence and uniqueness of strong solutions. For more details on the case $\alpha \geq 2$ we refer the reader to Remarks 4.3 and 5.3 below.

The situation is more delicate for flow behaviour exponents $\alpha \in(1,2)$. In this regime the operator $\mathcal{A}$ and the right-hand side $\mathcal{F}$ are only $(\alpha-1)$ Hölder continuous, whence there is no hope to obtain existence and uniqueness by Banach's fixed point theorem. Instead we use compactness to prove an abstract existence result for quasilinear parabolic problems of fourth order with Hölder continuous coefficients in the spirit of [4, Thm. 12.1]. More precisely, the proof exploits the a-priori estimates and the smoothing properties of the corresponding abstract linear equation to obtain a solution for the quasilinear problem by a fixed-point argument.

Finally we apply this result to obtain existence of solutions to (1.1) in (fractional) Sobolev spaces as well as in (little) Hölder spaces.

In order to prove uniqueness of solutions to (1.1), we explicitly retain its divergence form. Indeed, we use the special structure of the equation to obtain uniqueness by energy methods. This idea has already been used in the pioneering work [8] of BERNIS \& FRIEDMAN on the (Newtonian) thin-film equation.

We close this introduction by briefly outlining the organisation of this work. 
A derivation of the evolution problem is reviewed in Sect. 2. In Sect. 3 we recall an abstract well-posedness result of AMANN [3] for linear parabolic problems. Section 4 is concerned with an existence result for abstract quasilinear parabolic equations.

In Sect. 5 this result is applied to the non-Newtonian thin-film equation for all $\alpha>1$ and in the regime of fractional Sobolev and little Hölder spaces, respectively. Uniqueness of strong solutions to the non-Newtonian thin-film equation for flow behaviour exponents $\alpha \in(1,2)$ is proved in Sect. 6 . There we also show that constants are the only possible steady state solutions of (1.1). Finally, Sect. 7 contains a result on the maximal existence time of solutions to (1.1).

\section{Derivation of the non-Newtonian thin-film problem for an Ellis shear-thinning fluid}

For the convenience of the reader in this section we review the derivation of (1.1).

Lubrication approximation. Starting from the non-Newtonian NavierStokes equations with no-slip boundary condition, the lubrication approximation [20,34] leads - under the assumption of a positive film height - to the system

$$
\begin{aligned}
& 2-p_{x}+\left(\mu\left(\left|v_{z}\right|\right) v_{z}\right)_{z}=0 \quad \text { in } \Lambda \\
& p_{z}=0 \quad \text { in } \Lambda \\
& v_{x}+w_{z}=0 \quad \text { in } \Lambda \\
& v=w=0 \quad \text { on } z=0 \\
& p=-\sigma u_{x x} \quad \text { on } z=u(t, x) \\
& v_{z}=0 \quad \text { on } z=u(t, x) \\
& u_{t}+v u_{x}=w \quad \text { on } z=u(t, x)
\end{aligned}
$$

of dimensionless equations for the velocity field $(v, w)=(v(t, x, z), w(t, x, z))$, the pressure $p=p(t, x, z)$ and the film height $u=u(t, x)$. Here, $\sigma>0$ denotes the constant surface tension and $\mu: \mathbb{R} \rightarrow \mathbb{R}$ the fluid's viscosity. The fluid domain $\Lambda$ is defined by

$$
\Lambda:=\left\{(x, z) \in \mathbb{R}^{2} ; 0<x<l \text { and } 0<z<u(t, x)\right\} .
$$

Integrating $(2.1)_{2}$ from $z$ to $u$ and using the boundary condition $(2.1)_{5}$, we obtain

$$
p(x, z)=p(x, u)=-\sigma u_{x x}, \quad x \in \Omega .
$$

Moreover, an integration of $(2.1)_{1}$ from $z$ to $u$, together with the boundary condition $(2.1)_{6}$, yields

$$
\mu\left(\left|v_{z}\right|\right) v_{z}=\sigma u_{x x x}(u-z), \quad(x, z) \in \Lambda .
$$

SheAR-THinning RHEOlOGy: Ellis CONStitutive LAW. As for instance in $[5,43]$, we use the Ellis constitutive law to describe the fluid's shear-thinning 
rheology. We thus introduce the shear stress $\tau: \mathbb{R} \rightarrow \mathbb{R}$ implicitly by the relation

$$
s=\frac{1}{\mu_{0}}\left(1+\left|\frac{\tau(s)}{\tau_{*}}\right|^{\alpha-1}\right) \tau(s)
$$

and set

$$
\mu(s)=\frac{\tau(s)}{s} .
$$

Here, $\mu_{0}$ denotes the viscosity at zero shear stress and $\tau_{*}$ is the shear stress at which the viscosity is reduced by a factor $1 / 2$. Shear-thinning fluids are characterised by an apparent viscosity that decreases with increasing shear stress. For $\alpha>1$ and $\tau_{*} \in(0, \infty)$, the shear-thinning behaviour is reflected by the Ellis law. Moreover, it is observed in polymeric systems that, at rather low and/or rather high shear rates, the viscosity approaches a Newtonian plateau. For most polymers and polymer solutions $\alpha$ varies from 1 to 2 (see i.e. $[7,32]$ ). This Newtonian plateau may not be reflected by Ostwald-de Waele (pure power-law) fluids, which gives rise to the use of other models, as for instance the Ellis law. Observe that in $(2.3)-(2.4)$ the Newtonian plateau may be recovered for $\alpha=1$ or $1 / \tau_{*} \rightarrow 0$.

Using (2.3) and (2.4) in (2.2) leads to the equation

$$
v_{z}(x, z)=\frac{\sigma}{\mu_{0}} u_{x x x}(u-z)+\frac{\sigma^{\alpha}}{\mu_{0} \tau_{*}^{\alpha-1}}\left|u_{x x x}\right|^{\alpha-1} u_{x x x}(u-z)^{\alpha}, \quad(x, z) \in \Lambda,
$$

whence the horizontal velocity is given by

$$
\begin{aligned}
v(x, z)= & \frac{\sigma}{\mu_{0}} u_{x x x}\left(u z-\frac{z^{2}}{2}\right) \\
& -\frac{\sigma^{\alpha}}{(\alpha+1) \mu_{0} \tau_{*}^{\alpha-1}}\left|u_{x x x}\right|^{\alpha-1} u_{x x x}\left((u-z)^{\alpha+1}-u^{\alpha+1}\right), \quad(x, z) \in \Lambda .
\end{aligned}
$$

For $x \in(0, l)$, this finally yields

$$
\int_{0}^{u} v(x, z) d z=\frac{\sigma}{3 \mu_{0}} u_{x x x} u^{3}+\frac{\sigma^{\alpha}}{(\alpha+2) \mu_{0} \tau_{*}^{\alpha-1}}\left|u_{x x x}\right|^{\alpha-1} u_{x x x} u^{\alpha+2} .
$$

Hence, the evolution Eq. (2.1) 7 for the film's height $u$ reads

$$
u_{t}+a\left(u^{3}\left[1+\left|b u u_{x x x}\right|^{\alpha-1}\right] u_{x x x}\right)_{x}=0, \quad t>0, x \in \Omega,
$$

where the constants $a$ and $b$ are given by

$$
a:=\frac{\sigma}{3 \mu_{0}} \text { and } \quad b:=\left(\frac{3}{\alpha+2}\right)^{\frac{1}{\alpha-1}} \frac{\sigma}{\tau_{*}} .
$$

\section{A well-posedness result for abstract linear parabolic initial value problems}

This section is concerned with the solvability of linear parabolic evolution problems. We mainly recall a basic well-posedness and regularity result for linear parabolic Cauchy problems. We start by introducing some notations and requirements. 
In the following let $E_{0}$ and $E_{1}$ be Banach spaces. We write

$$
E_{1} \underset{d}{\hookrightarrow} E_{0} \quad \text { and } \quad E_{1} \underset{c}{\hookrightarrow} E_{0}
$$

if the continuous embedding of $E_{1}$ in $E_{0}$ is in addition dense, respectively compact. We say that $\left(E_{0}, E_{1}\right)$ is a densely injected Banach couple if $E_{1} \underset{d}{\hookrightarrow} E_{0}$. Given a densely injected Banach couple $\left(E_{0}, E_{1}\right)$ and $\theta \in(0,1)$, we denote by $(\cdot, \cdot)_{\theta}$ and $[\cdot, \cdot]_{\theta}$ a ${ }^{1}$ real, respectively the complex interpolation functor and set $E_{\theta}=\left(E_{0}, E_{1}\right)_{\theta}$, respectively $E_{\theta}=\left[E_{0}, E_{1}\right]_{\theta}$ with norm $\|\cdot\|_{E_{\theta}}$. It is well-known [3, Thm. I.2.11.1] that for a densely injected Banach couple $\left(E_{1}, E_{0}\right)$ we have

$$
E_{\alpha} \underset{d}{\hookrightarrow} E_{\beta}, \quad 0 \leq \beta<\alpha \leq 1
$$

Similarly, if $E_{1} \underset{c}{\hookrightarrow} E_{0}$, then

$$
E_{\alpha} \underset{c}{\hookrightarrow} E_{\beta}, \quad 0 \leq \beta<\alpha \leq 1 .
$$

Now let $\left(E_{0}, E_{1}\right)$ be a densely injected Banach couple and let $T>0$ be given. For each $t \in[0, T]$ let $\mathcal{A}(t)$ be a linear operator in $E_{0}$ with domain $E_{1}$ and let $\mathcal{F}:[0, T] \rightarrow E_{0}$. We consider the linear Cauchy problem

$$
\left\{\begin{array}{l}
\dot{u}+\mathcal{A}(t) u=\mathcal{F}(t), \quad t \in(0, T) \\
u(0)=0 .
\end{array}\right.
$$

We call (3.1) parabolic, if $-\mathcal{A}(t)$ is for each $t \in[0, T]$ the infinitesimal generator of a strongly continuous analytic semigroup on $E_{0}$, in symbols $\mathcal{A} \in \mathcal{H}\left(E_{1} ; E_{0}\right)$. We equip $\mathcal{H}\left(E_{1} ; E_{0}\right)$ with the operator norm $\|\cdot\|_{\mathcal{L}\left(E_{1} ; E_{0}\right)}$.

To derive estimates we need a quantitative description of $\mathcal{H}\left(E_{1}, E_{0}\right)$. Given $\kappa \geq 1$ and $\omega>0$, we write

$$
A \in \mathcal{H}\left(E_{1}, E_{0}, \kappa, \omega\right),
$$

if $\omega+A$ is an isomorphism from $E_{1}$ to $E_{0}$, and

$$
\kappa^{-1} \leq \frac{\|\lambda+A\|_{E_{0}}}{\mid \lambda\|x\|_{E_{0}}+\|x\|_{E_{1}}} \leq \kappa, \quad \text { whenever } x \in E_{1} \backslash\{0\}, \quad \operatorname{Re} \lambda \geq \omega .
$$

It holds that [3, Thm. I.1.2.2]

$$
\mathcal{H}\left(E_{1}, E_{0}\right)=\bigcup_{\substack{\kappa \geq 1 \\ \omega>0}} \mathcal{H}\left(E_{1}, E_{0}, \kappa, \omega\right) .
$$

We say that $u$ is a solution of (3.1) (in $E_{0}$ ) if the following conditions are satisfied:

(i) $u \in C\left([0, T] ; E_{0}\right) \cap C^{1}\left((0, T] ; E_{0}\right)$;

(ii) $u(0)=u_{0} \in E_{0}$ and $u(t) \in E_{1}$ for $t \in(0, T]$;

(iii) the differential equation $(3.1)_{1}$ holds in $(0, T)$.

We recall the following fundamental well-posedness and regularity result for linear parabolic Cauchy problems [3, Thm. II.1.2.1 and Thm. II.5.3.1].

\footnotetext{
${ }^{1}$ We say 'a' real interpolation functor $(\cdot, \cdot)_{\theta}$ since in our setting the real interpolation functor $(\cdot, \cdot)_{\theta, p}$ is admissible for all arbitrary parameters $p \in[1, \infty)$.
} 
Theorem 3.1. Let $\left(E_{0}, E_{1}\right)$ be a densely injected Banach couple and suppose that

$$
\mathcal{F} \in C^{\rho}\left([0, T] ; E_{0}\right) \quad \text { and } \quad \mathcal{A} \in C^{\rho}\left([0, T] ; \mathcal{H}\left(E_{1} ; E_{0}\right)\right)
$$

for some $\rho \in(0,1)$. Then the following holds true:

(i) If $u_{0} \in E_{0}$, then the linear Cauchy problem (3.1) possesses a unique solution

$$
u \in C^{\rho}\left((0, T) ; E_{1}\right) \cap C^{1+\rho}\left((0, T) ; E_{0}\right) \cap C\left([0, T] ; E_{0}\right) .
$$

(ii) If $u_{0} \in E_{1}$, then (3.1) possesses a unique solution

$$
u \in C\left([0, T] ; E_{1}\right) \cap C^{1}\left([0, T] ; E_{0}\right) .
$$

(iii) If $\alpha \in(0,1)$ and $u_{0} \in E_{\alpha}$, then the unique solution in (i) satisfies in addition

$$
u \in C\left([0, T] ; E_{\alpha}\right) .
$$

Let now $0 \leq \beta \leq \alpha<1$ and $\mathcal{F} \in L_{\infty, \text { loc }}\left([0, T] ; E_{0}\right)$, and assume that there exists a constant $C>0$ such that

$$
\mathcal{A} \in C^{\rho}\left([0, T] ; \mathcal{L}\left(E_{1} ; E_{0}\right)\right) \quad \text { with }[\mathcal{A}(\cdot)]_{C^{\rho}\left([0, T] ; \mathcal{L}\left(E_{1} ; E_{0}\right)\right)} \leq C .
$$

Moreover, assume that there are constants $\omega>0, \kappa \geq 1$ and $\sigma \in \mathbb{R}$ such that

$$
\sigma+\mathcal{A} \in C\left([0, T] ; \mathcal{H}\left(E_{1}, E_{0}, \kappa, \omega\right)\right) .
$$

Then we have in addition that,

(iv) if $u_{0} \in E_{\alpha}$, then $u \in C^{\alpha-\beta}\left([0, T] ; E_{\beta}\right)$ with

$\|u(t)-u(s)\|_{E_{\beta}} \leq C(t-s)^{\alpha-\beta} e^{\nu T}\left(\left\|u_{0}\right\|_{E_{\alpha}}+\|\mathcal{F}\|_{L_{\infty}\left([0, T] ; E_{0}\right)}\right), \quad t, s \in[0, T]$.

The constants $C, \nu>0$ do not depend on $T$.

A proof of this theorem can be found in the book [3]. While part (i) and part (iv) are due to AmAnN, part (ii) goes back to SobOLEVSKII [38] and TANABE [42]. Moreover, there is an analogous result by ACQUistaPACE \& TERRENI [2] which is proved by different methods. As regards assertion (iii), note that $\mathcal{A}([0, T])$ is a compact subset of $\mathcal{H}\left(E_{1}, E_{0}\right)$. Thus, by Amann [3, Cor. I.1.3.2] there exist $\kappa \geq 1$ and $\omega>0$ such that $A([0, T]) \subset H\left(E_{1}, E_{0}, \kappa, \omega\right)$. Now (3.5) follows from [3, Thm. II.5.3.1], applied with $\beta=\alpha$.

\section{Abstract existence theorem for the quasilinear problem}

In this section we prove an existence result for abstract quasilinear parabolic Cauchy problems, based on the theory for linear parabolic problems. This result will later be applied to the non-Newtonian thin-film equation in the setting of fractional Sobolev spaces and (little) Hölder spaces. problem

As in Sect. 3, let $T>0$ be given and consider the quasilinear Cauchy

$$
\left\{\begin{array}{l}
\dot{u}+\mathcal{A}(u) u=\mathcal{F}(u), \quad t \in(0, T) \\
u(0)=u_{0},
\end{array}\right.
$$


where $\mathcal{A}$ and $\mathcal{F}$ are Hölder continuous in an appropriate sense, to be specified below.

The existence result for (4.1) is deduced from the uniform a-priori estimates for the corresponding linear equation and an application of the following fixed-point theorem [21, Cor. 11.2].

Theorem 4.1. Let $X$ be a Banach space. Let $B \subset X$ be closed, convex and not empty. If $S: B \rightarrow B$ is continuous and compact, then $S$ has a fixed point.

It is worthwhile to mention again that with Theorem 4.1 we may obtain an existence result for (4.1) by compactness of the solution operator for the corresponding linear problem. Since we do not require Lipschitz continuity we can in general not expect to get uniqueness.

Theorem 4.2. Suppose that $\left(E_{0}, E_{1}\right)$ is a densely injected Banach couple and that the injection is in addition compact. Let $0<\beta<\alpha \leq 1$ and $\sigma \in(0, \alpha-\beta)$. Let $\mu \in(0,1)$ and assume that the maps

$$
\begin{aligned}
& \mathcal{A}: E_{\beta} \rightarrow \mathcal{H}\left(E_{1} ; E_{0}\right), \\
& \mathcal{F}: E_{\beta} \rightarrow E_{0}
\end{aligned}
$$

are $\mu$-Hölder continuous on all balls $B_{\beta}(0, R)$ in $E_{\beta}$. Then, for each $R^{\prime}>0$ there exist a positive time $T>0$ with the following property. If $u_{0} \in E_{\alpha}$ and $\left\|u_{0}\right\|_{E_{\alpha}} \leq R^{\prime}$, then the quasilinear initial value problem (4.1) possesses a solution

$$
u \in C^{\mu \sigma}\left((0, T] ; E_{1}\right) \cap C^{1+\mu \sigma}\left((0, T] ; E_{0}\right) \cap C\left([0, T] ; E_{\alpha}\right) .
$$

Moreover, for all $\alpha^{\prime} \in(\beta+\sigma, \alpha)$ the solution satisfies

$$
u \in C^{\sigma}\left([0, T] ; E_{\alpha^{\prime}-\sigma}\right) \cap C^{\alpha^{\prime}-\beta}\left([0, T] ; E_{\beta}\right) .
$$

Proof. Fix $\alpha, \beta$ and $R^{\prime}$. We use a fixed-point argument in a suitable ball in the space $C^{\rho}\left([0, T] ; E_{\beta}\right)$ for a suitable $\rho>0$. The argument uses the estimate (3.7). Thus, we start with the following observation. There exist $\omega>0, \kappa \geq 1$ and $r_{0}>0$ such that for all $w \in E_{\beta}$ we have the following implication:

$$
\left\|u_{0}\right\|_{E_{\alpha}} \leq R^{\prime} \quad \text { and } \quad\left\|w-u_{0}\right\|_{E_{\beta}} \leq r_{0} \quad \Longrightarrow \mathcal{A}(w) \in \mathcal{H}\left(E_{1}, E_{0}, \kappa, \omega\right) .
$$

To see this, note first that the set $K=\left\{u_{0} \in E_{\alpha}:\left\|u_{0}\right\|_{E_{\alpha}} \leq R^{\prime}\right\}$ is compact in $E_{\beta}$. Hence $\mathcal{A}(K)$ is a compact set in $\mathcal{H}\left(E_{1} ; E_{0}\right)$. By $(3.3)$ and a simple perturbation argument, see [3, Thm. I.1.3.1], this implies that there exist an open set $V$ and $\omega>0, \kappa \geq 1$ such that $\mathcal{A}(K) \subset V \subset \mathcal{H}\left(E_{1}, E_{0}, \kappa, \omega\right)$. Let $U$ be the preimage $U=\mathcal{A}^{-1}(V)$. Then $U$ is open in $E_{\beta}$ and $K \subset U$. Since $K$ is compact, it follows that $U$ contains a $2 r_{0}$ neighbourhood of $K$ for some $r_{0}>0$. Thus, (4.4) holds.

Now let $u_{0} \in E_{\alpha}$ such that $\left\|u_{0}\right\|_{E_{\alpha}} \leq R^{\prime}$. By $\bar{u}_{0}$ we denote the constant extension of $u_{0}$ on $[0, T]$. We set up a fixed-point problem, suitable for an application of Theorem 4.1, as follows. We set

$$
X:=C^{\sigma}\left([0, T] ; E_{\beta}\right) \quad \text { and } \quad \bar{B}:=\bar{B}\left(\bar{u}_{0}, r_{0}\right):=\left\{v \in X ;\left\|v-\bar{u}_{0}\right\|_{X} \leq r_{0}\right\} .
$$


Given $v \in \bar{B}$, the regularity assumptions on $\mathcal{A}$ and $\mathcal{F}$ imply

$$
\mathcal{A}(v(\cdot)) \in C^{\mu \sigma}\left([0, T] ; \mathcal{H}\left(E_{1} ; E_{0}\right)\right) \quad \text { and } \quad \mathcal{F}(v(\cdot)) \in C^{\mu \sigma}\left([0, T] ; E_{0}\right) .
$$

Furthermore, for $v \in \bar{B}$ we have

$$
\begin{aligned}
{[\mathcal{A}(v(\cdot))]_{C^{\mu \sigma}\left([0, T] ; \mathcal{L}\left(E_{1} ; E_{0}\right)\right)} } & \leq[\mathcal{A}]_{C^{\mu}\left(\bar{B}_{\beta}\left(0, R^{\prime}+r_{0}\right) ; \mathcal{L}\left(E_{1} ; E_{0}\right)\right)}[v]_{C^{\sigma}\left([0, T] ; E_{\beta}\right)}^{\mu} \\
& \leq[\mathcal{A}]_{C^{\mu}\left(\bar{B}_{\beta}\left(0, R^{\prime}+r_{0}\right) ; \mathcal{L}\left(E_{1} ; E_{0}\right)\right)}\left[v-\bar{u}_{0}\right]_{C^{\sigma}\left([0, T] ; E_{\beta}\right)}^{\mu} \\
& \leq[\mathcal{A}]_{C^{\mu}\left(\bar{B}_{\beta}\left(0, R^{\prime}+r_{0}\right) ; \mathcal{L}\left(E_{1} ; E_{0}\right)\right)} r_{0}^{\mu},
\end{aligned}
$$

i.e. a uniform bound of the $\mu \sigma$-Hölder norm of $\mathcal{A}$ on $[0, T]$. Moreover, recalling that $u_{0} \in \bar{B}_{\alpha}\left(0, R^{\prime}\right) \subset E_{\alpha}$ by assumption, we deduce from (4.4) the existence of some $\kappa \geq 1$ and $\omega>0$ such that

$$
\mathcal{A}(v(t)) \in \mathcal{H}\left(E_{1}, E_{0}, \kappa, \omega\right), \quad t \in[0, T],
$$

for all $v \in \bar{B}$. Finally, observe that for all $v \in \bar{B}$ we have the estimate

$$
\begin{aligned}
\|\mathcal{F}(v)\|_{L_{\infty}\left([0, T] ; E_{0}\right)} & \leq\left\|\mathcal{F}\left(u_{0}\right)\right\|_{E_{0}}+[\mathcal{F}]_{C^{\mu}\left(\bar{B}_{\beta}\left(0, R^{\prime}+r_{0}\right) ; E_{0}\right)}\left\|v-\bar{u}_{0}\right\|_{C^{\sigma}\left([0, T] ; E_{\beta}\right)}^{\mu} \\
& \leq\left\|\mathcal{F}\left(u_{0}\right)\right\|_{E_{0}}+[\mathcal{F}]_{C^{\mu}\left(\bar{B}_{\beta}\left(0, R^{\prime}+r_{0}\right) ; E_{0}\right)} r_{0}^{\mu} .
\end{aligned}
$$

Thus, we are in a position to apply Theorem 3.1 to deduce existence of a unique solution $u=S v$ of the linear evolution problem

$$
\left\{\begin{array}{l}
\dot{u}+\mathcal{A}(v(t)) u=\mathcal{F}(v(t)), \quad t \in(0, T] \\
u(0)=u_{0}
\end{array}\right.
$$

in the sense that $u=S v$ satisfies

$$
u \in C^{\mu \sigma}\left((0, T] ; E_{1}\right) \cap C^{1+\mu \sigma}\left((0, T] ; E_{0}\right) \cap C\left([0, T] ; E_{\alpha}\right) .
$$

Let now $\alpha^{\prime} \in(\beta+\sigma, \alpha)$ and note that $u_{0} \in E_{\alpha} \hookrightarrow E_{\alpha^{\prime}}$. Then the solution enjoys in addition the regularity

$$
u \in C^{\sigma}\left([0, T] ; E_{\alpha^{\prime}-\sigma}\right) \cap C^{\alpha^{\prime}-\beta}\left([0, T] ; E_{\beta}\right),
$$

and by (3.7) we have the estimates

$$
\begin{aligned}
& \|u\|_{C\left([0, T] ; E_{\alpha}\right)}+[u]_{C^{\sigma}\left([0, T] ; E_{\alpha^{\prime}-\sigma}\right)}+[u]_{C^{\alpha^{\prime}-\beta}\left([0, T] ; E_{\beta}\right)} \\
& \quad \leq C e^{\nu T}\left(\left\|u_{0}\right\|_{E_{\alpha}}+\|\mathcal{F}(v)\|_{L_{\infty}\left([0, T] ; E_{0}\right)}\right),
\end{aligned}
$$

where $\nu \geq 0$ is independent of $T$. In view of the uniform estimate $(4.5)$ on $\mathcal{F}$, the solution $u=S v$ satisfies in fact the uniform estimate

$$
\|u\|_{C\left([0, T] ; E_{\alpha}\right)}+[u]_{C^{\sigma}\left([0, T] ; E_{\alpha^{\prime}-\sigma}\right)}+[u]_{C^{\alpha^{\prime}-\beta}\left([0, T] ; E_{\beta}\right)} \leq C\left(R^{\prime}, r_{0}\right) e^{\nu T},
$$

where the constants $C$ and $\nu$ are independent of $T$.

In order to deduce from Theorem 4.1 the existence of a fixed point $u=$ $S u \in X$, we are thus left with verifying that

(i) $S: \bar{B} \rightarrow X$ is continuous;

(ii) $S: \bar{B} \rightarrow X$ is compact;

(iii) $S$ preserves the ball $\bar{B}$. 
(i) Continuity of $S$. From the linear theory we know that $S$ maps the space $\bar{B}$ continuously into

$$
Y:=C^{\sigma}\left([0, T] ; E_{\alpha^{\prime}-\sigma}\right) \cap C^{\alpha^{\prime}-\beta}\left([0, T] ; E_{\beta}\right) .
$$

Since $\alpha^{\prime}-\sigma>\beta$, we have $E_{\alpha^{\prime}-\sigma} \hookrightarrow E_{\beta}$ and hence continuity of the embedding

$$
C^{\sigma}\left([0, T] ; E_{\alpha^{\prime}-\sigma}\right) \hookrightarrow C^{\sigma}\left([0, T] ; E_{\beta}\right) .
$$

(ii) Compactness of $S$. We know from the linear theory that $S$ maps $\bar{B}$ to $Y$. We show that $Y$ is compactly embedded into $X$. Since $\alpha^{\prime}-\sigma>\beta$, it follows from the compactness of the embedding $E_{\alpha^{\prime}-\sigma} \underset{c}{\hookrightarrow} E_{\beta}$ and the Arzelà-Ascoli theorem that

$$
C^{\sigma}\left([0, T] ; E_{\alpha^{\prime}-\sigma}\right) \underset{c}{\hookrightarrow} C\left([0, T] ; E_{\beta}\right) .
$$

Hence, $S$ is a compact operator from $\bar{B}$ to $C\left([0, T] ; E_{\beta}\right)$. In view of the interpolation estimate

$$
[u]_{C^{\sigma}\left([0, T] ; E_{\beta}\right)} \leq\|u\|_{C\left([0, T] ; E_{\beta}\right)}^{1-\theta}[u]_{C^{\alpha^{\prime}-\beta}\left([0, T] ; E_{\beta}\right)}^{\theta} \quad \text { with } \theta=\frac{\sigma}{\alpha^{\prime}-\beta},
$$

we see that $S$ is even a compact operator from $\bar{B}$ to $X$.

(iii) $S(\bar{B}) \subset \bar{B}$. To deduce that $S$ has a fixed point, we are left with verifying that, for sufficiently small $T>0$, the operator $S$ maps the ball $\bar{B} \subset X$ into itself. Recall from (4.7) that, given $v \in \bar{B}$, we obtain the estimate

$$
[u]_{C^{\alpha^{\prime}-\beta}\left([0, T] ; E_{\beta}\right)} \leq C\left(R^{\prime}, r_{0}\right) e^{\nu T}
$$

for the solution $u=S v$ of the linear problem (4.6). This implies on the one hand that

$$
\left\|u-\bar{u}_{0}\right\|_{C\left([0, T] ; E_{\beta}\right)} \leq C\left(R^{\prime}, r_{0}\right) T^{\alpha^{\prime}-\beta} e^{\nu T}
$$

and on the other hand (recall that $\sigma<\alpha^{\prime}-\beta$ )

$$
\left[u-\bar{u}_{0}\right]_{C^{\sigma}\left([0, T] ; E_{\beta}\right)} \leq T^{\alpha^{\prime}-\beta-\sigma}\left[u-\bar{u}_{0}\right]_{C^{\alpha^{\prime}-\beta}\left([0, T] ; E_{\beta}\right)} \leq C\left(R^{\prime}, r_{0}\right) T^{\alpha^{\prime}-\beta-\sigma} e^{\nu T} .
$$

Combining (4.9) and (4.10), we find that

$$
\left\|u-\bar{u}_{0}\right\|_{C^{\sigma}\left([0, T] ; E_{\beta}\right)} \leq C\left(R^{\prime}, r_{0}\right)\left(T^{\alpha^{\prime}-\beta-\sigma}+T^{\alpha^{\prime}-\beta}\right) e^{\nu T} .
$$

Thus, for sufficiently small $T>0$, the right-hand side of this inequality is less than or equal $r_{0}$, which proves that $S(\bar{B}) \subset \bar{B}$.

Remark 4.3. Recall that our motivation to prove this abstract result is to prove existence of solutions to the non-Newtonian thin-film equation (1.1) in different function spaces. As mentioned in the introduction, for flow behaviour exponents $\alpha \geq 2$ for instance the results of Amman [4, Thm. 12.1] and Eidel'man [14, III.4.6.3] are applicable and provide existence and uniqueness at the same time. On the other hand there seem to be no abstract results available for $\alpha \in(1,2)$. Roughly speaking, the reason for this qualitative difference is that in the case $\alpha \in(1,2)$ the Nemitskii operator associated to the function $f(x)=|x|^{\alpha-1}$ is only $(\alpha-1)$-Hölder continuous, while it is Lipschitz continuous for $\alpha \geq 2$. See also Remark 5.3 for more details. The Lipschitz continuity 
allows one to get existence and uniqueness by a contraction argument. However, concerning existence of solutions to the non-Newtonian thin-film equation (1.1) we cover the case of flow behaviour exponents $\alpha \in(1,2)$ by applying our abstract existence result Theorem 4.2, while we deduce uniqueness from energy estimates that use the structure of the particular equation.

In the remainder of this section we prove a result on the maximal existence time of solutions. We use the usual continuation argument to obtain a contradiction. However, some care is required in the formulation of the result since solutions may not be unique. We fix $u_{0} \in E_{\alpha}$ and set

$$
\begin{aligned}
\bar{T}:= & \sup \left\{T>0 ; \exists \text { solution } u \in C^{1}\left((0, T] ; E_{0}\right)\right. \\
& \left.\cap C\left((0, T] ; E_{1}\right) \cap C\left([0, T] ; E_{\alpha}\right) \text { of }(4.1) \text { with } u(0)=u_{0}\right\},
\end{aligned}
$$

and prove that the following holds true.

Theorem 4.4. Let $0<\beta<\alpha \leq 1$ and $u_{0} \in E_{\alpha}$. Let $\bar{T}$ be defined by (4.11) and assume that $\bar{T}<\infty$. Furthermore, let $\gamma \in(\beta, \alpha]$ and $R>0$. Then there exists a time $T_{R}(\gamma) \in(0, \bar{T})$ with the following property. If $T \geq T_{R}(\gamma)$ and if

$$
u \in C\left((0, T] ; E_{1}\right) \cap C^{1}\left((0, T] ; E_{0}\right) \cap C\left([0, T] ; E_{\alpha}\right)
$$

is a solution of (4.1) with $u(0)=u_{0}$, then

$$
\|u(t)\|_{E_{\gamma}}>R \text { for all } t \geq T_{R}(\gamma) .
$$

Proof. Fix $\gamma \in(\beta, \alpha]$. Let $T_{0}=T_{0}(\gamma, R)$ be the existence time in Theorem 4.2, with $\alpha$ replaced by $\gamma$ and $R^{\prime}$ replaced by $R$. We claim that the assertion of the theorem holds with

$$
T_{R}(\gamma)=\bar{T}-\frac{T_{0}}{2}
$$

Indeed, assume that there exists a solution $u \in C\left([0, T] ; E_{\alpha}\right)$ with

$$
\|u(\bar{t})\|_{E_{\gamma}} \leq R \quad \text { and } \quad T \geq \bar{t} \geq \bar{T}-\frac{T_{0}}{2} .
$$

Then by Theorem 4.2 there exists a solution

$$
U \in C\left(\left[0, T_{0}\right] ; E_{\gamma}\right) \cap C^{\frac{\gamma-\beta}{2}}\left(\left[0, T_{0}\right] ; E_{\beta}\right)
$$

with initial value $U(0)=u(\bar{t})$. Here we used Theorem 4.2 with $\alpha=\gamma$ and $\alpha^{\prime}=\frac{\beta+\gamma}{2}$. Thus,

$$
\mathcal{A} \circ U \in C^{\rho}\left(\left[0, T_{0}\right] ; \mathcal{H}\left(E_{1} ; E_{0}\right)\right) \quad \text { and } \quad \mathcal{F} \circ U \in C^{\rho}\left(\left[0, T_{0}\right] ; E_{0}\right)
$$

with $\rho=\mu \frac{\gamma-\beta}{2}$. Moreover, $U(0)=u(\bar{t}) \in E_{1}$. Therefore, the linear theory gives $U \in C^{1}\left(\left[0, T_{0}\right] ; E_{0}\right) \cap C\left(\left[0, T_{0}\right] ; E_{1}\right)$. Now define

$$
\tilde{u}(t)= \begin{cases}u(t), & 0 \leq t<\bar{t} \\ U(t-\bar{t}), & \bar{t} \leq t \leq \bar{t}+T_{0} .\end{cases}
$$

Then $\tilde{u} \in C\left(\left[0, \bar{t}+T_{0}\right] ; E_{\alpha}\right) \cap C\left(\left(0, \bar{t}+T_{0}\right] ; E_{1}\right)$. The equation

$$
\dot{\tilde{u}}+\mathcal{A}(\tilde{u}) \tilde{u}=\mathcal{F}(\tilde{u})
$$


holds in $(0, \bar{t})$ and in $\left(\bar{t}, \bar{t}+T_{0}\right)$. Since $\tilde{u} \in C\left(\left(0, \bar{t}+T_{0}\right] ; E_{1}\right)$, it follows that $\dot{\tilde{u}}$ can be uniquely continued at $t=\bar{t}$ to a continuous function with values in $E_{0}$. Indeed, thanks to the continuity of $\mathcal{A}$ and $\mathcal{F}$ we have for $t>\bar{t}$

$$
\begin{aligned}
0 & =\lim _{t \searrow \bar{t}} \partial_{t}^{+} U(t-\bar{t})+\mathcal{A}(U(t-\bar{t})) U(t-\bar{t})-\mathcal{F}(U(t-\bar{t})) \\
& =\partial_{t}^{+} U(0)+\mathcal{A}(U(0)) U(0)-\mathcal{F}(U(0)) \\
& =\partial_{t}^{+} \tilde{u}(\bar{t})+\mathcal{A}(\tilde{u}(\bar{t})) \tilde{u}(\bar{t})-\mathcal{F}(\tilde{u}(\bar{t})) \text { in } E_{0}
\end{aligned}
$$

and for $t<\bar{t}$

$$
\begin{aligned}
0 & =\lim _{t \nearrow \bar{t}} \partial_{t}^{-} u(t)+\mathcal{A}(u(t)) u(t)-\mathcal{F}(u(t)) \\
& =\partial_{t}^{-} \tilde{u}(\bar{t})+\mathcal{A}(\tilde{u}(\bar{t})) \tilde{u}(\bar{t})-\mathcal{F}(\tilde{u}(\bar{t})) \text { in } E_{0} .
\end{aligned}
$$

Thus, $\tilde{u} \in C\left(\left[0, \bar{t}+T_{0}\right] ; E_{\alpha}\right) \cap C\left(\left(0, \bar{t}+T_{0}\right] ; E_{1}\right) \cap C^{1}\left(\left(0, \bar{t}+T_{0} ; E_{0}\right)\right.$ and $\tilde{u}$ is a solution of $\dot{\tilde{u}}+\mathcal{A}(\tilde{u}) \tilde{u}=\mathcal{F}(\tilde{u})$ on $\left(0, \bar{t}+T_{0}\right)$. Since $\bar{t}+T_{0} \geq \bar{T}+\frac{T_{0}}{2}>\bar{T}$, this contradicts the definition of $\bar{T}$.

\section{Existence of solutions to the non-Newtonian thin-film equation}

In this section we apply the abstract existence result Theorem 4.2 to the nonNewtonian thin-film equation

$$
\begin{cases}u_{t}+a\left(u^{3}\left[1+\left|b u u_{x x x}\right|^{\alpha-1}\right] u_{x x x}\right)_{x}=0, & t>0, x \in \Omega \\ u_{x}=u_{x x x}=0, & t>0, x \in \partial \Omega \\ u(0, \cdot)=u_{0}(\cdot), & x \in \Omega .\end{cases}
$$

We first introduce some notation. Using the identity

$$
\begin{aligned}
\left(u^{3}\left[1+\left|b u u_{x x x}\right|^{\alpha-1}\right] u_{x x x}\right)_{x}= & u^{3}\left(1+\alpha\left|b u u_{x x x}\right|^{\alpha-1}\right) u_{x x x x} \\
& +3 u^{2}\left(1+\left|\tilde{b} u u_{x x x}\right|^{\alpha-1}\right) u_{x} u_{x x x},
\end{aligned}
$$

where $\tilde{b}=\sigma / \tau_{*}$, we may rewrite (5.1) in the following way in non-divergence form:

$$
\begin{cases}u_{t}+A\left(u, u_{x}, u_{x x}, u_{x x x}\right) u_{x x x x}=F\left(u, u_{x}, u_{x x}, u_{x x x}\right), & t>0, x \in \Omega \\ u_{x}=u_{x x x}=0, & t>0, x \in \partial \Omega \\ u(0, \cdot)=u_{0}(\cdot), & x \in \Omega,\end{cases}
$$

where

$$
A:(0, \infty) \times \mathbb{R}^{3} \longrightarrow(0, \infty), \quad A\left(z_{0}, z_{1}, z_{2}, z_{3}\right)=a z_{0}^{3}\left(1+\alpha\left|b z_{0} z_{3}\right|^{\alpha-1}\right),
$$

and

$$
F: \mathbb{R}^{4} \longrightarrow \mathbb{R}, \quad F\left(z_{0}, z_{1}, z_{2}, z_{3}\right)=-3 z_{0}^{2}\left(1+\left|\tilde{b} z_{0} z_{3}\right|^{\alpha-1}\right) z_{1} z_{3} .
$$

are $(\alpha-1)$-Hölder continuous. Moreover, given a function $v:[0, T] \times \Omega \rightarrow \mathbb{R}$, we write

$$
V(t, x)=\left(v, v_{x}, v_{x x}, v_{x x x}\right)
$$


and we use the abbreviations

$$
A_{V}(t)=(A \circ V)(t) \text { and } F_{V}(t)=(F \circ V)(t) .
$$

Constructing solutions of (5.1), respectively (5.2), naturally involves the following two challenges. First, to be able to apply the abstract existence result Theorem 4.2 we have to reformulate (5.2) as an abstract quasilinear Cauchy problem. In other words we have to choose a suitable Banach space $E_{0}$ in which we study the problem and we have to define the differential operator $\mathcal{A}$ properly. This means in particular that we have to define $\mathcal{A}$ such that its domain

$$
D(\mathcal{A})=\left\{u \in E_{0} ; \mathcal{A}(v) u \in E_{0} \forall v \in E_{\beta} ; u_{x}=u_{x x x}=0 \text { on } \partial \Omega\right\}
$$

incorporates the first-order and third-order Neumann boundary conditions. Of course we need that $\mathcal{A}$ generates an analytic semigroup on $E_{0}$ and that $\mathcal{A}$ and $\mathcal{F}$ satisfy the required regularity properties. Moreover, $\left(E_{1}, E_{0}\right)$ has to be a densely and compactly injected Banach couple.

The second challenge we have to deal with is that the non-Newtonian thin-film equation is reasonable for positive film heights $u$ only. Hence, in order to apply Theorem 4.2 we extend problem (5.2) in a way such that for positive initial data solutions of the extended problem coincide for a short time with solutions of the original problem.

To tackle the latter challenge we extend the coefficient map $A$ to a globally defined, locally Hölder continuous function as follows. For $v^{+}=\max (v, 0)$, we first introduce the map

$$
\bar{A}: \mathbb{R}^{4} \longrightarrow[0, \infty), \quad \bar{A}\left(z_{0}, z_{1}, z_{2}, z_{3}\right)=A\left(z_{0}^{+}, z_{1}, z_{2}, z_{3}\right) .
$$

Note that the function $v \mapsto v^{+}$is locally Lipschitz continuous and hence, we still have $\bar{A} \in C_{\text {loc }}^{\alpha-1}\left(\mathbb{R}^{4}\right)$. Finally, let $\varepsilon>0$ be given. To ensure parabolicity of the coefficient map, we set

$$
\bar{A}_{\varepsilon}: \mathbb{R}^{4} \longrightarrow(\varepsilon / 2, \infty), \quad \bar{A}_{\varepsilon}\left(z_{0}, z_{1}, z_{2}, z_{3}\right)=\max \left(\bar{A}\left(z_{0}, z_{1}, z_{2}, z_{3}\right), \varepsilon / 2\right) .
$$

Summarising, we have that the maps $A, \bar{A}, \bar{A}_{\varepsilon}$ and $F$ are locally $(\alpha-1)$-Hölder continuous on $\mathbb{R}^{4}$, in symbols

$$
\bar{A}_{\varepsilon} \in C_{\mathrm{loc}}^{\alpha-1}\left(\mathbb{R}^{4} ;(0, \infty)\right) \quad \text { and } \quad F \in C_{\mathrm{loc}}^{\alpha-1}\left(\mathbb{R}^{4} ;(0, \infty)\right),
$$

(and analogously for $A$ and $\bar{A}$ ). That is, for all $z, z^{\prime} \in \mathbb{R}^{4}$ with $|z|,\left|z^{\prime}\right| \leq R$ they satisfy

$$
\left|\bar{A}_{\varepsilon}(z)-\bar{A}_{\varepsilon}\left(z^{\prime}\right)\right| \leq C_{R}\left|z-z^{\prime}\right|^{\alpha-1} \quad \text { and } \quad\left|F(z)-F\left(z^{\prime}\right)\right| \leq C_{R}\left|z-z^{\prime}\right|^{\alpha-1}
$$

(and analogously for $A$ and $\bar{A}$ ). As above, we finally introduce the notation

$$
\bar{A}_{\varepsilon, V}(t)=\left(\bar{A}_{\varepsilon} \circ V\right)(t) .
$$

The corresponding global version of (5.2) then reads

$$
\begin{cases}u_{t}+\bar{A}_{\varepsilon}\left(u, u_{x}, u_{x x}, u_{x x x}\right) u_{x x x x}=F\left(u, u_{x}, u_{x x}, u_{x x x}\right), & t>0, x \in \Omega \\ u_{x}=u_{x x x}=0, & t>0, x \in \partial \Omega \\ u(0, \cdot)=u_{0}(\cdot), & x \in \Omega .\end{cases}
$$


The task of setting up an appropriate framework for the abstract Cauchy problem in terms of function spaces is addressed in the following two subsections.

\subsection{Solutions to (5.1) in fractional Sobolev spaces}

In this section we study the problem of existence of solutions to (5.1), respectively (5.2), which are Hölder continuous in time and take values in Sobolev spaces of fractional order. Note that we consider only the case in which $\Omega \subset \mathbb{R}$ is a bounded interval.

For $k \in \mathbb{N}$ and $p \in[1, \infty)$ we denote by $W_{p}^{k}(\Omega)$ the usual Sobolev spaces with norm

$$
\|v\|_{W_{p}^{k}(\Omega)}=\left(\sum_{j=0}^{k}\left\|\partial^{j} v\right\|_{L_{p}(\Omega)}^{p}\right)^{1 / p}
$$

We then put

$$
[v]_{W_{p}^{s}(\Omega)}=\int_{\Omega} \int_{\Omega} \frac{|v(x)-v(z)|^{p}}{|x-z|^{1+s p}} d x d z, \quad 1 \leq p<\infty, 0<s<1,
$$

and define the Sobolev-Slobodeckii or fractional Sobolev spaces by

$$
W_{p}^{s}(\Omega)=\left\{v \in W_{p}^{[s]}(\Omega) ;\|v\|_{W_{p}^{s}(\Omega)}<\infty\right\}, \quad 1 \leq p<\infty, s \in \mathbb{R}_{+} \backslash \mathbb{N},
$$

where

$$
\|v\|_{W_{p}^{s}(\Omega)}=\left(\|v\|_{W_{p}^{[s]}(\Omega)}^{p}+\left[\partial^{[s]} v\right]_{W_{p}^{s-[s]}(\Omega)}^{p}\right)^{1 / p}, \quad 1 \leq p<\infty, s \in \mathbb{R}_{+} \backslash \mathbb{N} .
$$

Here, $[s]$ denotes the largest integer smaller than or equal to $s$.

We now recall some important properties of these spaces, which are necessary to guarantee that we are in the setting of Theorem 4.2. It is wellknown that, for $-\infty<s_{0}<s_{1}<\infty$ and $0<\rho<1$, the space $W_{p}^{s}(\Omega), s=$ $(1-\rho) s_{0}+\rho s_{1}$, is the complex interpolation space between $W_{p}^{s_{1}}(\Omega)$ and $W_{p}^{s_{0}}(\Omega)$, in symbols

$$
W_{p}^{s}(\Omega)=\left[W_{p}^{s_{0}}(\Omega), W_{p}^{s_{1}}(\Omega)\right]_{\rho}
$$

In order to take the (Neumann) boundary conditions of (5.1), respectively (5.2), into account, we further introduce the Banach spaces

$$
W_{p, B}^{4 \rho}(\Omega)= \begin{cases}\left\{v \in W_{p}^{4 \rho}(\Omega) ; v_{x}=v_{x x x}=0 \text { on } \partial \Omega\right\}, & 3+\frac{1}{p}<4 \rho \leq 4 \\ \left\{v \in W_{p}^{4 \rho}(\Omega) ; v_{x}=0 \text { on } \partial \Omega\right\}, & 1+\frac{1}{p}<4 \rho \leq 3+\frac{1}{p} \\ W_{p}^{4 \rho}(\Omega), & 0 \leq 4 \rho \leq 1+\frac{1}{p} .\end{cases}
$$

For $4 \rho \in(0,4) \backslash\{1+1 / p, 3+1 / p\}$, the spaces $W_{p, B}^{4 \rho}(\Omega)$ are closed linear subspaces of $W_{p}^{4 \rho}(\Omega)$ and satisfy the interpolation property

$$
W_{p, B}^{4 \rho}(\Omega)=\left(L_{p}(\Omega), W_{p, B}^{4}(\Omega)\right)_{\rho, p}, \quad 1<p<\infty .
$$

We can now apply the abstract existence result Theorem 4.2 to the nonNewtonian thin-film equation (5.2). More precisely, we prove the following theorem on the existence of solutions in Sobolev spaces of fractional order. 
Theorem 5.1. Let $p \in(1, \infty)$ and $1 / p<s<r<1$. Moreover, let $\sigma=\frac{3+s}{4}$ and $\rho=\frac{3+r}{4}$. Then, given an initial film height $u_{0} \in W_{p, B}^{4 \rho}(\Omega)$ such that $u_{0}(x)>0$ for all $x \in \bar{\Omega}$, for each $\alpha \in(1,2)$ there exists a positive $T>0$ and a solution $u$ of (5.1) on $[0, T]$ in the sense that

$$
\begin{aligned}
u & \in C\left([0, T], W_{p, B}^{4 \rho}(\Omega)\right) \cap C^{\rho}\left([0, T], L_{p}(\Omega)\right) \cap C\left((0, T], W_{p, B}^{4}(\Omega)\right) \\
& \cap C^{1}\left((0, T], L_{p}(\Omega)\right)
\end{aligned}
$$

and

$$
u(t, x)>0, \quad(t, x) \in[0, T] \times \bar{\Omega} .
$$

In order to prove Theorem 5.1 we have to verify that the conditions of the abstract result Theorem 4.2 are satisfied. To this end, we make the choice

$$
E_{0}=L_{p}(\Omega), \quad E_{1}=W_{p, B}^{4}(\Omega) .
$$

For this choice it is well-known that

$$
W_{p, B}^{4}(\Omega) \underset{d}{\hookrightarrow} L_{p}(\Omega) \quad \text { and } \quad W_{p, B}^{4}(\Omega) \underset{c}{\hookrightarrow} L_{p}(\Omega), \quad 1<p<\infty .
$$

According to $(5.7)$ we denote by $E_{\rho}=W_{p, B}^{4 \rho}(\Omega), 4 \rho \in(0,4) \backslash\{1+1 / p, 3+1 / p\}$, the respective real interpolation spaces. Then we also have (see for instance [3, Thm. I.2.11.1])

$$
W_{p, B}^{4 \rho}(\Omega) \underset{d}{\hookrightarrow} W_{p, B}^{4 \sigma}(\Omega) \quad \text { and } \quad W_{p, B}^{4 \rho}(\Omega) \underset{c}{\hookrightarrow} W_{p, B}^{4 \sigma}(\Omega), \quad 0 \leq \sigma<\rho \leq 1 .
$$

Based on that we view the evolution Eq. $(5.2)_{1}$ in non-divergence form as an abstract quasilinear Cauchy problem in the following way. Let $p \in(1, \infty)$ and $s>1 / p$. For $v \in W_{p, B}^{4 \sigma}(\Omega)$ with $\sigma=\frac{3+s}{4}$, such that $v(x)>0$ for all $x \in \bar{\Omega}$, we associate to (5.2) the linear differential operator

$$
\mathcal{A}(v(t)) \in \mathcal{L}\left(W_{p, B}^{4}(\Omega) ; L_{p}(\Omega)\right), \quad \mathcal{A}(v(t)) u:=A_{V}(t) \partial_{x}^{4} u,
$$

of fourth order. Then, with

$$
\mathcal{F}(v(t))=-3 a\left(v^{2} v_{x} v_{x x x}+\tilde{b}^{\alpha-1} v^{\alpha+1} v_{x}\left|v_{x x x}\right|^{\alpha-1} v_{x x x}\right),
$$

we rewrite (5.2) as

$$
\left\{\begin{array}{l}
\dot{u}+\mathcal{A}(u) u=\mathcal{F}(u), \quad t>0 \\
u(0)=u_{0} .
\end{array}\right.
$$

Similarly, we rewrite the extended problem (5.6) as

$$
\left\{\begin{array}{l}
\dot{u}+\overline{\mathcal{A}}_{\varepsilon}(u) u=\mathcal{F}(u), \quad t>0 \\
u(0)=u_{0},
\end{array}\right.
$$

where

$$
\overline{\mathcal{A}}_{\varepsilon}(v(t)) \in \mathcal{L}\left(W_{p, B}^{4}(\Omega) ; L_{p}(\Omega)\right), \quad \overline{\mathcal{A}}_{\varepsilon}(v(t)) u:=\bar{A}_{\varepsilon, V}(t) \partial_{x}^{4} u .
$$

In the following lemmas we study the relevant regularity properties of the differential operator $\overline{\mathcal{A}}_{\varepsilon}$ and the right-hand side $\mathcal{F}$, introduced in (5.12), respectively (5.9). 
Lemma 5.2. Given $p \in(1, \infty)$ and $1 / p<s<1$, let $\sigma=\frac{3+s}{4}$. Then for all flow behaviour exponents $\alpha \in(1,2)$ the mappings

$$
\overline{\mathcal{A}}_{\varepsilon}: W_{p, B}^{4 \sigma}(\Omega) \longrightarrow \mathcal{L}\left(W_{p, B}^{4}(\Omega) ; L_{p}(\Omega)\right) \text { and } \mathcal{F}: W_{p, B}^{4 \sigma}(\Omega) \longrightarrow L_{p}(\Omega),
$$

are $(\alpha-1)$-Hölder continuous on bounded balls in the sense that

$$
\begin{aligned}
\left\|\overline{\mathcal{A}}_{\varepsilon}(v)-\overline{\mathcal{A}}_{\varepsilon}(w)\right\|_{\mathcal{L}\left(W_{p, B}^{4}(\Omega), L_{p}(\Omega)\right)} & \leq C_{R}\|v-w\|_{W_{p, B}^{4 \sigma}(\Omega)}^{\alpha-1} \quad \text { and } \\
\|\mathcal{F}(v)-\mathcal{F}(w)\|_{L_{p}(\Omega)} & \leq C_{R}\|v-w\|_{W_{p, B}^{4 \sigma}(\Omega)}^{\alpha-1}
\end{aligned}
$$

for all $v, w \in W_{p, B}^{4 \sigma}(\Omega)$ with $\|v\|_{W_{p, B}^{4 \sigma}(\Omega)},\|w\|_{W_{p, B}^{4 \sigma}(\Omega)} \leq R$.

Proof. This follows from (5.5).

We can now prove the main result of this section.

Proof of Theorem 5.1. (i) Existence. Let $p \in(1, \infty)$ and $1 / p<s<r<1$. Then put $\sigma=\frac{3+s}{4}$ and $\rho=\frac{3+r}{4}$. Suppose that $u_{0} \in W_{p, B}^{4 \rho}$ such that

$$
\left\|u_{0}\right\|_{W_{p, B}^{4 \rho}(\Omega)}<R^{\prime} \quad \text { and } \quad u_{0}(x) \geq 2\left(\frac{\varepsilon}{2 a}\right)^{1 / 3}>0 \quad \forall x \in \bar{\Omega}
$$

where $\varepsilon, R^{\prime}>0$ are fixed. We first apply Theorem 4.2 to show that problem (5.11) possesses a solution for some time $T_{\varepsilon}>0$. To this end, note that in view of Lemma 5.2 we have the required Hölder continuity of the operator $\overline{\mathcal{A}}_{\varepsilon}$ and the right-hand side;

$$
\begin{aligned}
\overline{\mathcal{A}}_{\varepsilon} & : W_{p, B}^{4 \sigma}(\Omega) \longrightarrow \mathcal{L}\left(W_{p, B}^{4}(\Omega) ; L_{p}(\Omega)\right), \\
\mathcal{F} & : W_{p, B}^{4 \sigma}(\Omega) \longrightarrow L_{p}(\Omega) .
\end{aligned}
$$

Note that here we used that $\overline{\mathcal{A}}_{\varepsilon}$ is the composition of $\mathcal{A}$ with two Lipschitz continuous maps. Moreover, recall that the choice of $\sigma=\frac{3+s}{p}$ with $s>1 / p$ implies that $W_{p, B}^{4 \sigma}(\Omega) \hookrightarrow C(\bar{\Omega})$, whence for $v \in W_{p, B}^{4 \sigma}(\Omega)$

$$
A_{V}=a v^{3}\left(1+\alpha\left|b v v_{x x x}\right|^{\alpha-1}\right) \in C(\bar{\Omega})
$$

and thus finally

$$
\bar{A}_{\varepsilon}=\max \left(A\left((v)_{+}, v_{x}, v_{x x}, v_{x x x}\right), \varepsilon / 2\right) \in C(\bar{\Omega}) .
$$

In addition, the principal symbol $a_{\varepsilon}(x, \xi)$ of the operator $\mathcal{A}_{\varepsilon}(v)$ satisfies the uniform Legendre-Hadamard condition

$$
\operatorname{Re}\left(a_{\varepsilon}(x, \xi) \eta \mid \eta\right) \geq \frac{\varepsilon}{2}(i \xi)^{4} \eta^{2}>0
$$

for $(x, \xi) \in \bar{\Omega} \times\{-1,1\}$ and $\eta \in \mathbb{R} \backslash\{0\}$. Thus, $-\overline{\mathcal{A}}_{\varepsilon}(v)$ with the given boundary conditions is normally elliptic in the sense of [4, Example 4.3(d)]. Thanks to [4, Thm. $4.1 \&$ Rem.4.2(b)] we conclude that

$$
\overline{\mathcal{A}}_{\varepsilon}(v) \in \mathcal{H}\left(W_{p, B}^{4}(\Omega), L_{p}(\Omega)\right),
$$

i.e. $-\overline{\mathcal{A}}_{\varepsilon}(v)$ generates an analytic semigroup on $L_{p}(\Omega)$. In virtue of (5.13) and (5.14) we may eventually apply Theorem 4.2 to conclude that there exists a positive time $T_{\varepsilon}$ and a solution

$$
u_{\varepsilon} \in C\left([0, T] ; W_{p, B}^{4 \rho}(\Omega)\right) \cap C^{\nu}\left([0, T] ; W_{p, B}^{4 \sigma}(\Omega)\right),
$$


with $\nu \in(0, \rho-\sigma)$, to the extended problem (5.11).

(ii) Positivity. As above, we denote by $\bar{u}_{0}$ the constant extension of $u_{0}$ on $[0, T]$. Now, if $u_{0}(x) \geq 2\left(\frac{\varepsilon}{2 a}\right)^{1 / 3}$ for all $x \in \bar{\Omega}$, we find that

$$
\min _{t \in[0, T]} u_{\varepsilon}(t, x) \geq 2\left(\frac{\varepsilon}{2 a}\right)^{1 / 3}-C T^{\nu}
$$

for all $x \in \bar{\Omega}$. Hence,

$$
u_{\varepsilon}(t, x)>\left(\frac{\varepsilon}{2 a}\right)^{1 / 3}, \quad(t, x) \in[0, T] \times \bar{\Omega},
$$

for $T<\left(\frac{1}{C}\left(\frac{\varepsilon}{2 a}\right)^{1 / 3}\right)^{1 / \nu}$.

(iii) It remains to show that the solution $u_{\varepsilon}$ is - at least for a short timealso a solution to (5.10). Indeed, by (5.15) we obtain

$$
\min _{t \in[0, T]} A_{U_{\varepsilon}}(t) \geq \min _{t \in[0, T]} a u_{\varepsilon}(t)^{3} \geq \frac{\varepsilon}{2}
$$

for $T<\left(\frac{1}{C}\left(\frac{\varepsilon}{2 a}\right)^{1 / 3}\right)^{1 / \nu}$. This implies that there exists a positive time $T^{*}$ such that

$$
A_{U_{\varepsilon}}(t)=\bar{A}_{\varepsilon, U_{\varepsilon}}(t), \quad t \in\left[0, T^{*}\right],
$$

and hence $u_{\varepsilon}$ does also solve the original problem $(5.10)$ on $\left[0, T^{*}\right]$. This completes the proof.

Remark 5.3. It is worthwhile to discuss again the qualitative differences originating from the different values of the flow behaviour exponent $\alpha$. Note that for $\alpha \geq 2$ Lemma 5.2 can be improved to Lipschitz continuity in the appropriate norms. Indeed, recall that $W_{p}^{s}(\Omega)$ is a Banach algebra and that the Nemitskii operator induced by the function $f(z)=|z|^{\alpha-1}$ acts on $W_{p}^{s}(\Omega)$ for $\alpha \geq 2$, see for instance [35, Thm. 4.6.4/2], respectively [35, Thm. 5.4.3/1]. Together with the inequality

$$
\left.|| z\right|^{\beta}-|y|^{\beta}\left|\leq C_{\beta}\left(|z|^{\beta-1}+|y|^{\beta-1}\right)\right| z-y \mid, \quad \beta \geq 1,
$$

this makes the following calculation possible. For $p \in(1, \infty)$ and $s>1 / p$ we have

$$
\begin{aligned}
&\left\|\left(v^{\alpha+2}\left|v_{x x x}\right|^{\alpha-1}-w^{\alpha+2}\left|w_{x x x}\right|^{\alpha-1}\right) u_{x x x x}\right\|_{L_{p}(\Omega)} \\
& \leq C\left(\|\left.\left(v^{\alpha+2}-w^{\alpha+2}\right)\left|v_{x x x}\right|^{\alpha-1}\right|_{L_{p}(\Omega)}\right. \\
&\left.+\|\left. w^{\alpha+2}\left(\left|v_{x x x}\right|^{\alpha-1}-\left|w_{x x x}\right|^{\alpha-1}\right)\right|_{L_{p}(\Omega)}\right)\|u\|_{W_{p}^{4}(\Omega)} \\
& \leq C_{\alpha}\left(\left\|\left|v_{x x x}\right|^{\alpha-1}||_{W_{p}^{s}(\Omega)}|| v^{\alpha+2}-w^{\alpha+2}\right\|_{L_{\infty}(\Omega)}\right. \\
&\left.+\left\|w^{\alpha+2}||_{W_{p}^{s}(\Omega)}||\left|v_{x x x}\right|^{\alpha-1}-\left|w_{x x x}\right|^{\alpha-1}\right\|_{L_{\infty}(\Omega)}\right)\|u\|_{W_{p}^{4}(\Omega)} \\
& \leq C_{\alpha}\left(\| v-\left.w\right|_{W_{p}^{3+s}(\Omega)}+\left[\left\|\left|v_{x x x}\right|^{\alpha-2}\right\|_{L_{\infty}(\Omega)}\right.\right. \\
&\left.\left.+\left.\left\|\left|w_{x x x}\right|^{\alpha-2}\right\|\right|_{L_{\infty}(\Omega)}\right]|| v_{x x x}-w_{x x x} \|_{W_{p}^{s}(\Omega)}\right)\|u\|_{W_{p}^{4}(\Omega)} \\
& \leq C_{\alpha}\|v-w\|_{W_{p}^{3+s}(\Omega)} .
\end{aligned}
$$


This means that for $\alpha \geq 2$ one can even prove that $\mathcal{A} \in \operatorname{Lip}\left(W_{p}^{4 \sigma}(\Omega)\right.$; $\left.\mathcal{H}\left(W_{p}^{4}(\Omega), L_{p}(\Omega)\right)\right)$, where $\sigma=(3+s) / 4$. A similar calculation shows that $\mathcal{F} \in \operatorname{Lip}\left(W_{p}^{4 \sigma}(\Omega) ; L_{p}(\Omega)\right)$. Hence, for $\alpha \geq 2$ we are in the regime of $[4$, Thm. 12.1] which gives existence and uniqueness of solutions to (5.1) in the sense of Theorem 5.1.

\subsection{Solutions of (5.1) in (little) Hölder spaces}

This section is devoted to the existence of classical solutions to the nonNewtonian thin-film equation (5.1), respectively (5.2). More precisely, we apply our abstract Theorem 4.2 in the setting of (little) Hölder spaces. Note again that we study the one-dimensional thin-film equation.

As in Sect. 5.1, we start by introducing the relevant notation and function spaces. Let $\Omega \subset \mathbb{R}$ be an open and bounded interval. For $k \in \mathbb{N}$ and $\rho \in(0,1)$ we define the usual Hölder spaces by

$$
\begin{gathered}
C^{\rho}(\bar{\Omega})=\left\{v \in C(\bar{\Omega}) ;[v]_{C^{\rho}(\bar{\Omega})}=\sup _{x, z \in \bar{\Omega}, x \neq z} \frac{|v(x)-v(z)|}{|x-z|^{\rho}}<\infty\right\} \text { with } \\
\|v\|_{C^{\rho}(\bar{\Omega})}=\|v\|_{C(\bar{\Omega})}+[v]_{C^{\rho}(\bar{\Omega})}
\end{gathered}
$$

and

$$
\begin{aligned}
C^{k+\rho}(\bar{\Omega}) & =\left\{v \in C^{k}(\bar{\Omega}) ;\left[v^{(k)}\right]_{C^{\rho}(\bar{\Omega})}<\infty\right\} \text { with } \\
\|v\|_{C^{k+\rho}(\bar{\Omega})} & =\|v\|_{C^{k}(\bar{\Omega})}+\left[v^{(k)}\right]_{C^{\rho}(\bar{\Omega})} .
\end{aligned}
$$

We further introduce the so-called little-Hölder spaces

$$
h^{\rho}(\bar{\Omega})=\left\{v \in C^{\rho}(\bar{\Omega}) ; \lim _{\varepsilon \rightarrow 0} \sup _{x, z \in \bar{\Omega} ; 0<|x-z|<\varepsilon} \frac{|v(x)-v(z)|}{|x-z|^{\rho}}=0\right\}
$$

and

$$
h^{k+\rho}(\bar{\Omega})=\left\{v \in C^{k+\rho}(\bar{\Omega}) ; \lim _{\varepsilon \rightarrow 0} \sup _{x, z \in \bar{\Omega} ; 0<|x-z|<\varepsilon} \frac{\left|v^{(k)}(x)-v^{(k)}(z)\right|}{|x-z|^{\rho}}=0\right\} .
$$

We recall some important properties of these spaces. The space $h^{\rho}(\bar{\Omega})$ is a closed subspace of $C^{\rho}(\bar{\Omega})$ and hence a Banach space. If $0<\sigma<1$, then $h^{\sigma}(\bar{\Omega})$ is the closure of $C^{\rho}(\bar{\Omega})$ in $C^{\sigma}(\bar{\Omega})$ for all $\rho \in(\sigma, \infty]$. Furthermore, for $0 \leq s_{0}<s_{1}$ and $0<\rho<1$, the space $h^{s}(\bar{\Omega}), s=(1-\rho) s_{0}+\rho s_{1}$ is the real interpolation space between $C^{s_{1}}(\bar{\Omega})$ and $C^{s_{0}}(\bar{\Omega})$, in symbols

$$
h^{s}(\bar{\Omega})=\left(C^{s_{0}}(\bar{\Omega}), C^{s_{1}}(\bar{\Omega})\right)_{\rho}, \quad s \notin \mathbb{N}, 0 \leq s_{0}<s_{1} .
$$

In order to take the first-order and third-order Neumann boundary conditions of problem (5.1) into account, we further introduce for $\rho \in(0,1]$ the spaces

$$
h_{B}^{4 \rho}(\bar{\Omega})= \begin{cases}\left\{v \in h^{4 \rho}(\bar{\Omega}) ; v_{x}=v_{x x x}=0 \text { on } \partial \Omega\right\}, & 3 \leq 4 \rho \leq 4 \\ \left\{v \in h^{4 \rho}(\bar{\Omega}) ; v_{x}=0 \text { on } \partial \Omega\right\}, & 1 \leq 4 \rho<3 \\ h^{4 \rho}(\bar{\Omega}), & 0<4 \rho<1\end{cases}
$$


For $4 \rho \in(0,4), 4 \rho \notin \mathbb{N}$, the spaces $h_{B}^{4 \rho}(\bar{\Omega})$ are closed linear subspaces of $h^{4 \rho}(\bar{\Omega})$. Thanks to [1, Thm. 2.3] they may be characterised as the real interpolation spaces between $C_{B}^{4}(\bar{\Omega})$ and $C(\bar{\Omega})$;

$$
h_{B}^{4 \rho}(\bar{\Omega})=\left(C_{B}^{4}(\bar{\Omega}), C(\bar{\Omega})\right)_{\rho}, \quad 4 \rho \notin \mathbb{N} .
$$

The main result of this section may now be formulated as follows.

Theorem 5.4. Let $3 / 4<\sigma<\rho \leq 1$. Then, given an initial film height $u_{0} \in$ $h_{B}^{4 \rho}(\bar{\Omega})$, such that $u_{0}(x)>0$ for all $x \in \bar{\Omega}$, for each $\alpha>1$ there exists a positive time $T>0$ and a solution $u$ of (5.1) on $[0, T]$ in the sense that

$u \in C\left([0, T] ; h_{B}^{4 \rho}(\bar{\Omega})\right) \cap C^{\rho}([0, T] ; C(\bar{\Omega})) \cap C\left((0, T] ; C_{B}^{4}(\bar{\Omega})\right) \cap C^{1}((0, T] ; C(\bar{\Omega}))$. If in addition $u_{0} \in C_{B}^{4}(\bar{\Omega})$, then

$$
C\left([0, T] ; C_{B}^{4}(\bar{\Omega})\right) \cap C^{1}([0, T] ; C(\bar{\Omega})) .
$$

In any case $u$ satisfies

$$
u(t, x)>0, \quad(t, x) \in[0, T] \times \bar{\Omega} .
$$

The Proof of Theorem 5.4 is similar to the one in the setting of fractional Sobolev spaces.

We verify that the conditions of the abstract result Theorem 4.2 are satisfied and identify

$$
E_{0}=C(\bar{\Omega}) \quad \text { and } \quad E_{1}=C_{B}^{4}(\bar{\Omega}) .
$$

For this choice it is well-known that

$$
C_{B}^{4}(\bar{\Omega}) \underset{d}{\hookrightarrow} C(\bar{\Omega}) \quad \text { and } \quad C_{B}^{4}(\bar{\Omega}) \underset{c}{\hookrightarrow_{c}} C(\bar{\Omega})
$$

Denoting by $E_{\rho}=h_{p, B}^{4 \rho}(\bar{\Omega})$ the respective interpolation spaces, we have (see for instance [3, Thm. I.2.11.1])

$$
h_{B}^{4 \rho}(\bar{\Omega}) \underset{d}{\hookrightarrow} h_{B}^{4 \sigma}(\bar{\Omega}) \quad \text { and } \quad h_{B}^{4 \rho}(\bar{\Omega}) \hookrightarrow_{c} h_{B}^{4 \sigma}(\bar{\Omega}), \quad 0 \leq \sigma<\rho \leq 1 .
$$

As before, we view the evolution Eq. $(5.2)_{1}$ in non-divergence form as an abstract quasilinear Cauchy problem. For $v \in h_{B}^{4 \sigma}(\Omega)$ with $\sigma=\frac{3+s}{4}$, such that $v(x)>0$ for all $x \in \bar{\Omega}$, we associate to (5.2) the linear differential operator

$$
\mathcal{A}(v(t)) \in \mathcal{L}\left(C_{B}^{4}(\bar{\Omega}) ; C(\bar{\Omega})\right), \quad \mathcal{A}(v(t)) u:=A_{V}(t) \partial_{x}^{4} u,
$$

of fourth order. Then, with

$$
\mathcal{F}(v(t))=-3 a\left(v^{2} v_{x} v_{x x x}+\tilde{b}^{\alpha-1} v^{\alpha+1} v_{x}\left|v_{x x x}\right|^{\alpha-1} v_{x x x}\right),
$$

we rewrite $(5.2)$ as

$$
\left\{\begin{array}{l}
\dot{u}+\mathcal{A}(u) u=\mathcal{F}(u), \quad t>0 \\
u(0)=u_{0} .
\end{array}\right.
$$

As in the Proof of Theorem 5.1, we first solve the extended problem and then prove that the solution also satisfies the original equations. 
Proof of Theorem 5.4. As in Lemma 5.2 one obtains that the right-hand side, considered as a map $\mathcal{F}: h_{B}^{4 \sigma}(\bar{\Omega}) \rightarrow C(\bar{\Omega})$, and the differential operator $\overline{\mathcal{A}}_{\varepsilon}$ : $h_{B}^{4 \sigma}(\bar{\Omega}) \rightarrow \mathcal{L}\left(C_{B}^{4}(\bar{\Omega}) ; C(\bar{\Omega})\right)$ are Hölder continuous on all balls in the sense that

$$
\begin{aligned}
& \|\mathcal{F}(v)-\mathcal{F}(w)\|_{C(\bar{\Omega})} \leq C_{R}\|v-w\|_{h_{B}^{4 \sigma}(\bar{\Omega})}^{\alpha-1} \text { and } \\
& \left\|\overline{\mathcal{A}}_{\varepsilon}(v)-\overline{\mathcal{A}}_{\varepsilon}(w)\right\|_{\mathcal{L}\left(C_{B}^{4}(\bar{\Omega}) ; C(\bar{\Omega})\right)} \leq C_{R}\|v-w\|_{h_{B}^{4 \sigma}(\bar{\Omega})}^{\alpha-1}
\end{aligned}
$$

for all $v, w \in h_{B}^{4 \sigma}(\bar{\Omega})$ with $\|v\|_{\left.h_{B}^{4 \sigma}(\bar{\Omega})\right)},\|w\|_{h_{B}^{4 \sigma}(\bar{\Omega})} \leq R$.

From $[40,41]$ we know that $-\overline{\mathcal{A}}_{\varepsilon}(v)$ generates, for each $v \in h_{B}^{4 \sigma}(\bar{\Omega})$, an analytic semigroup on $C(\bar{\Omega})$, i.e.

$$
\overline{\mathcal{A}}_{\varepsilon}(v) \in \mathcal{H}\left(C_{B}^{4}(\bar{\Omega}) ; C(\bar{\Omega})\right) .
$$

We obtain the assertion by following the lines of the proof of Theorem 5.1.

\section{Uniqueness of solutions to (5.1) for flow behaviour exponents $\alpha \in(1,2)$}

Recall from Sects. 5.1 and 5.2 that, for flow behaviour exponents $\alpha \geq 2$, we have Lipschitz continuity of the differential operator $\mathcal{A}$ as well as the righthand side $\mathcal{F}$. Thus, for $\alpha \geq 2$ we are in the setting of Eidel'man [14, Thm. III.4.6.3] and Amann [4, Thm. 12.1] and obtain uniqueness of solutions to (5.1) by a contraction argument.

For flow behaviour exponents $\alpha \in(1,2)$ we get existence of solutions to (5.1) in fractional Sobolev and little Hölder spaces, respectively, by compactness of the solution operator for the linear problem, c.f. Theorems 5.1 and 5.4.

In this section we prove uniqueness of solutions to (5.1) by deriving an energy inequality for which we use the special structure of the equation. More precisely, we extend the approach used in [8] for the Newtonian thin-film equation to prove that, for $\alpha \in(1,2)$, two positive strong solutions of (5.1) coincide if this is the case initially. For this purpose, observe that the energy

$$
E(u)=\frac{1}{2} \int_{\Omega}\left|u_{x}\right|^{2} d x
$$

decreases along smooth solutions of (5.1). Indeed, if $u$ is a smooth solution of (5.1), then

$$
\frac{d}{d t} E(u(t))=-\int_{\Omega} u_{x x} u_{t} d x=-a \int_{\Omega} u^{3}\left|u_{x x x}\right|^{2}+b^{\alpha-1} u^{\alpha+2}\left|u_{x x x}\right|^{\alpha+1} d x
$$

and hence

$$
\frac{1}{2} \int_{\Omega} u_{x}^{2}(t) d x+a \int_{0}^{T} \int_{\Omega} u^{3}\left|u_{x x x}\right|^{2}+b^{\alpha-1} u^{\alpha+2}\left|u_{x x x}\right|^{\alpha+1} d x d t=\frac{1}{2} \int_{\Omega}\left(u_{0}\right)_{x}^{2} d x .
$$

To justify the energy inequality (6.1) for solutions in our regularity class and to apply a similar argument to the difference of two solutions we use the following fact. 
Proposition 6.1. Suppose that $w_{1}, w_{2} \in C\left((0, T) ; W_{p^{\prime}, 0}^{1}(\Omega)\right) \cap C^{1}((0, T)$, $\left.W_{p}^{-1}(\Omega)\right)$. Then the map $t \mapsto\left\langle w_{1}(t), w_{2}(t)\right\rangle$ is differentiable in $(0, T)$ and

$$
\frac{d}{d t} \int_{\Omega} w_{1} w_{2} d x=\left\langle\frac{d}{d t} w_{1}, w_{2}\right\rangle+\left\langle\frac{d}{d t} w_{2}, w_{1}\right\rangle,
$$

where $\langle\cdot, \cdot\rangle$ denotes the dual pairing between $W_{p}^{-1}(\Omega)$ and $W_{p^{\prime}}^{1}(\Omega)$.

Proof. This follows by writing out the difference quotient and noting that $h^{-1}\left(w_{2}(t+h)-w_{2}(t)\right)$ is bounded in $W_{p}^{-1}(\Omega)$, while $w_{1}(t+h)-w_{1}(t)$ goes to to zero in $W_{p^{\prime}, 0}^{1}(\Omega)$, as $h \rightarrow 0$.

Proposition 6.1 guarantees in particular that the expression $\frac{d}{d t} E(u(t))$ is well-defined for solutions $u$ obtained by Theorem 5.1 in the fractional Sobolev space setting or by Theorem 5.4 in the little Hölder setting. This allows us to show the following uniqueness result.

Theorem 6.2. Let $\alpha>1$. Let $u$ and $v$ be two positive solutions of (5.1) on $[0, T]$, as in Theorem 5.1 or Theorem 5.4, emanating from the same initial value $u_{0}$, where $u_{0}(x)>0$ for all $x \in \bar{\Omega}$. Then $u=v$ on $[0, T]$.

Proof. We consider only solutions obtained by Theorem 5.1. The proof for solutions in the sense of Theorem 5.4 is the same. By the usual continuation argument it suffices to show that there exists a time $0<T_{*} \leq T$ such that $u=v$ on $\left[0, T_{*}\right)$. Since both $u$ and $v$ are positive as long as they exist, there is a $0<T_{0}<T$ and constants $c, C>0$ such that

$$
0<c \leq u(t, x), v(t, x) \leq C, \quad t \in\left[0, T_{0}\right], x \in \bar{\Omega} .
$$

For all $t \in\left(0, T_{0}\right)$ we may now extend the arguments of [8] in the following way.

We know that $(u-v) \in C\left((0, T] ; W_{p, B}^{4}(\Omega)\right) \cap C^{1}\left((0, T) ; L_{p}(\Omega)\right)$. Since $W_{p, B}^{4}(\Omega)$ embedds into $C^{3}(\bar{\Omega})$, in particular $(u-v)_{x} \in C\left((0, T] ; C^{2}(\bar{\Omega})\right)$ and $(u-v)_{x}=0$ on $\partial \Omega$. Thus, it follows from Proposition 6.1 that $t \mapsto \int_{\Omega}\left(u_{x}-\right.$ $\left.v_{x}\right)^{2} d x$ is differentiable in $(0, T)$ and

$$
\frac{d}{d t} \frac{1}{2} \int_{\Omega}\left(u_{x}-v_{x}\right)^{2} d x=\left\langle\left(u_{t}-v_{t}\right)_{x},(u-v)_{x}\right\rangle=-\int_{\Omega}\left(u_{t}-v_{t}\right)\left(u_{x x}-v_{x x}\right) d x .
$$

Using the equations for $u_{t}$ and $v_{t}$, integrating by parts once more, and using that $u_{x x x}=v_{x x x}=0$ on $\partial \Omega$ we get, after integration in time,

$$
\begin{aligned}
& \frac{1}{2} \int_{\Omega}\left(u_{x}(t)-v_{x}(t)\right)^{2} d x-\frac{1}{2} \int_{\Omega}\left(u_{x}(s)-v_{x}(s)\right)^{2} d x \\
& =-\int_{s}^{t} \int_{\Omega}\left(u^{3}\left|u_{x x x}\right|^{2}+b^{\alpha-1} u^{\alpha+2}\left|u_{x x x}\right|^{\alpha+1}-v^{3}\left|v_{x x x}\right|^{2}\right. \\
& \left.\quad+b^{\alpha-1} v^{\alpha+2}\left|v_{x x x}\right|^{\alpha+1}\right)\left(u_{x x x}-v_{x x x}\right) d x d \tau
\end{aligned}
$$

for all $0<s<t<T$. Since $4 \rho>3+\frac{1}{p}$, the space $W_{p, B}^{4 \rho}(\Omega)$ embedds into $C^{3}(\bar{\Omega})$ and we have $u, v \in C\left([0, T] ; C^{3}(\bar{\Omega})\right.$. Thus, we can easily pass to the limit $s \downarrow 0$ and conclude that (6.2) also holds for $s=0$. 
Using elementary manipulations of the integrands on the right-hand side and the fact that $u(0)=v(0)=u_{0}$, we deduce the following identity for the relative energy

$$
\begin{aligned}
\frac{1}{2} \int_{\Omega} & \left(u_{x}(t)-v_{x}(t)\right)^{2} d x \\
= & -a \int_{0}^{t} \int_{\Omega}\left|u_{x x x}-v_{x x x}\right|^{2} u^{3} d x d s \\
& -a \int_{0}^{t} \int_{\Omega}\left(u_{x x x}-v_{x x x}\right) v_{x x x}\left(u^{3}-v^{3}\right) d x d s \\
& -a b^{\alpha-1} \int_{0}^{t} \int_{\Omega}\left(u_{x x x}-v_{x x x}\right) u^{\alpha+2}\left(\left|u_{x x x}\right|^{\alpha-1} u_{x x x}\right. \\
& \left.-\left|v_{x x x}\right|^{\alpha-1} v_{x x x}\right) d x d s \\
& -a b^{\alpha-1} \int_{0}^{t} \int_{\Omega}\left(u_{x x x}-v_{x x x}\right)\left|v_{x x x}\right|^{\alpha-1} v_{x x x} \\
& \left(u^{\alpha+2}-v^{\alpha+2}\right) d x d s .
\end{aligned}
$$

Since $u$ is bounded away from zero by $c>0$, we may use the inequality (cf. $[12$, Lemma 4.4])

$\left(\left|u_{x x x}\right|^{\alpha-1} u_{x x x}-\left|v_{x x x}\right|^{\alpha-1} v_{x x x}\right)\left(u_{x x x}-v_{x x x}\right) \geq c_{\alpha}\left|u_{x x x}-v_{x x x}\right|^{\alpha+1}, \quad \alpha \geq 1$

in the third integral of the right-hand side of (6.3) to obtain

$$
\begin{aligned}
& \frac{1}{2} \int_{\Omega}\left(u_{x}(t)-v_{x}(t)\right)^{2} d x+a c^{3} \int_{0}^{t} \int_{\Omega}\left|u_{x x x}-v_{x x x}\right|^{2} d x d s \\
& \quad+c^{\alpha+2} c_{\alpha} \int_{0}^{t} \int_{\Omega}\left|u_{x x x}-v_{x x x}\right|^{\alpha+1} d x d s \\
& \leq a \int_{0}^{t} \int_{\Omega}\left|\left(u_{x x x}-v_{x x x}\right) v_{x x x}\left(u^{3}-v^{3}\right)\right| d x d s \\
& \quad+\left.a b^{\alpha-1} \int_{0}^{t} \int_{\Omega}\left|\left(u_{x x x}-v_{x x x}\right)\right| v_{x x x}\right|^{\alpha-1} v_{x x x}\left(u^{\alpha+2}-v^{\alpha+2}\right) \mid d x d s .
\end{aligned}
$$

Applying Young's (weighted) inequality to the remaining two integrals on the right-hand side, respectively, yields

$$
\begin{aligned}
\int_{0}^{t} \int_{\Omega}\left|\left(u_{x x x}-v_{x x x}\right) v_{x x x}\left(u^{3}-v^{3}\right)\right| d x d s \leq & \frac{c^{3}}{4} \int_{0}^{t} \int_{\Omega}\left|u_{x x x}-v_{x x x}\right|^{2} d x d s \\
& +\frac{1}{c^{3}} \int_{0}^{t} \int_{\Omega}\left|v_{x x x}\right|^{2}\left|u^{3}-v^{3}\right|^{2} d x d s
\end{aligned}
$$


and

$$
\begin{aligned}
b^{\alpha-1} & \left.\int_{0}^{t} \int_{\Omega}\left|\left(u_{x x x}-v_{x x x}\right)\right| v_{x x x}\right|^{\alpha-1} v_{x x x}\left(u^{\alpha+2}-v^{\alpha+2}\right) \mid d x d s \\
\leq & \frac{c^{3}}{4} \int_{0}^{t} \int_{\Omega}\left|u_{x x x}-v_{x x x}\right|^{2} d x d s \\
& +\frac{b^{2(\alpha-1)}}{c^{3}} \int_{0}^{t} \int_{\Omega}\left|v_{x x x}\right|^{2 \alpha}\left|u^{\alpha+2}-v^{\alpha+2}\right|^{2} d x d s .
\end{aligned}
$$

Hence, we find that

$$
\begin{aligned}
& \frac{1}{2} \int_{\Omega}\left(u_{x}(t)-v_{x}(t)\right)^{2} d x \leq \frac{a}{c^{3}} \int_{0}^{t} \int_{\Omega}\left|v_{x x x}\right|^{2}\left|u^{3}-v^{3}\right|^{2} \\
& \quad+b^{2(\alpha-1)}\left|v_{x x x}\right|^{2 \alpha}\left|u^{\alpha+2}-v^{\alpha+2}\right|^{2} d x d s .
\end{aligned}
$$

Recalling that both $u$ and $v$ are bounded above, using the elementary inequalities

$$
\left|u^{3}-v^{3}\right|^{2} \leq C(u, v)|u-v|^{2} \quad \text { and } \quad\left|u^{\alpha+2}-v^{\alpha+2}\right|^{2} \leq C(u, v)|u-v|^{2}
$$

and an $L_{1}-L_{\infty}$ Hölder estimate on $(0, t) \times \Omega$ yields

$$
\begin{aligned}
\sup _{s \in(0, t)} \int_{\Omega}\left(u_{x}(s, x)-v_{x}(s, x)\right)^{2} d x \leq & C \int_{0}^{t} \int_{\Omega}\left(\left|v_{x x x}\right|^{2}+\left|v_{x x x}\right|^{2 \alpha}\right)|u-v|^{2} d x d s \\
\leq & C\left(\int_{0}^{t} \int_{\Omega}\left(\left|v_{x x x}\right|^{2}+\left|v_{x x x}\right|^{2 \alpha}\right) d x d s\right) \| u \\
& -v \|_{L_{\infty}((0, t) \times \Omega)}^{2} .
\end{aligned}
$$

In virtue of the Sobolev embedding $H^{1}(\Omega) \hookrightarrow L_{\infty}(\Omega)$ we eventually arrive at

$$
\begin{aligned}
& \sup _{s \in(0, t)} \int_{\Omega}\left(u_{x}(s, x)-v_{x}(s, x)\right)^{2} d x \\
& \quad \leq C\left(\int_{0}^{t} \int_{\Omega}\left(\left|v_{x x x}\right|^{2}+\left|v_{x x x}\right|^{2 \alpha}\right) d x d s\right) \sup _{s \in(0, t)} \int_{\Omega}\left(u_{x}(s, x)-v_{x}(s, x)\right)^{2} d x .
\end{aligned}
$$

Choosing $t$ small enough we obtain $u_{x} \equiv v_{x}$ and hence the assertion.

We now prove that flat films are the only possible steady state solutions of (5.1).

Corollary 6.3. Let $u$ be a solution of (5.1) in the sense of Theorem 5.1 or Theorem 5.4 and assume that $u_{t}=0$ for $t=0$. Then $u$ is constant in space, $u=u_{*} \in \mathbb{R}_{>0}$.

Proof. The energy inequality (6.1) in connection with Proposition 6.1 implies that

$$
0=\frac{d}{d t} E(u)=-a \int_{\Omega} u^{3}\left|u_{x x x}\right|^{2}+b^{\alpha-1} u^{\alpha+2}\left|u_{x x x}\right|^{\alpha+1} d x,
$$

where the right-hand side is non-positive. Therefore, the positivity of $u_{*}$ implies that $\left(u_{*}\right)_{x x x}=0$. Thus, $\left(u_{*}\right)_{x x}$ is constant which in turn implies that $\left(u_{*}\right)_{x}$ is linear. Together with the Neumann boundary condition we obtain that $\left(u_{*}\right)_{x} \equiv$ 0 and finally that $u_{*} \equiv$ const. 


\section{Maximal time of existence}

In this section we characterise the maximal time of existence of solutions to the non-Newtonian thin-film equation, obtained by Theorem 5.1, respectively Theorem 5.4. For convenience we consider only solutions in fractional Sobolev spaces, i.e. solutions in the sense of Theorem 5.1. The argument may easily be adapted to the case of little Hölder functions.

In order to state the precise result we use the same notation

$$
p \in(1, \infty), \quad 1 / p<s<r<1, \quad \sigma=\frac{3+s}{4} \quad \text { and } \quad \rho=\frac{3+r}{4}
$$

as in Theorem 5.1. We then define, for $u_{0} \in W_{p, B}^{4 \rho}(\Omega)$ with $u_{0}(x)>0$ for all $x \in \bar{\Omega}$,

$$
\begin{aligned}
& \bar{T}=\sup \{T>0 \text {; there exists a solution } u \\
& \quad \text { of (5.1) in the sense of Theorem (5.1) }\} .
\end{aligned}
$$

It follows from the uniqueness result Theorem 6.2 that there exists a solution

$$
u \in C\left([0, \bar{T}) ; W_{p, B}^{4 \rho}(\Omega)\right) \cap C\left((0, \bar{T}) ; W_{p, B}^{4}(\Omega)\right) \cap C^{1}\left((0, \bar{T}) ; L_{p}(\Omega)\right) .
$$

We prove that solutions with a finite lifetime $\bar{T}<\infty$ do either converge to zero for some point in $\bar{\Omega}$ or they blow up in every $W_{p, B}^{4 \gamma}(\Omega)$-norm, where $\gamma \in(\sigma, 1]$.

Theorem 7.1. Suppose that $\bar{T}<\infty$. Then

$$
\liminf _{t \nearrow \bar{T}} \frac{1}{\min _{\bar{\Omega}} u(t)}+\|\left. u(t)\right|_{W_{p, B}^{4 \gamma}(\Omega)}=\infty
$$

for all $\gamma \in(\sigma, 1]$.

Proof. This follows from the standard continuation argument. Fix $\gamma \in(\sigma, \rho]$. Let $\bar{T}<\infty$ and assume by contradiction that (7.3) is false. Then there exist positive constants $r, R_{\gamma}>0$ and a sequence $\left(t_{n}\right)_{n}$ with $\lim _{n \rightarrow \infty} t_{n}=\bar{T}$ such that

$$
\min _{\bar{\Omega}} u\left(t_{n}\right) \geq r \quad \text { and } \quad\left\|u\left(t_{n}\right)\right\|_{W_{p, B}^{4 \gamma}(\Omega)} \leq R_{\gamma}, \quad n \in N .
$$

By Theorem 5.1 there exists a positive time $T=T\left(r, R_{\gamma}\right)>0$ (independent of $n$ ) such that for all $n \in N$ there exists a solution

$$
U_{n} \in C\left([0, T] ; W_{p, B}^{4 \gamma}(\Omega)\right) \cap C^{\frac{\gamma-\sigma}{2}}\left([0, T] ; W_{p, B}^{4 \sigma}(\Omega)\right) \cap C\left((0, T] ; W_{p, B}^{4}(\Omega)\right)
$$

with initial value $U_{n}(0)=u\left(t_{n}\right)$. Here we used Theorem 5.1 with $\rho=\gamma$ and $\rho^{\prime}=\frac{\sigma+\rho}{2}$, whence

$$
\mathcal{A} \circ U_{n} \in C^{\nu}\left([0, T] ; \mathcal{H}\left(W_{p, B}^{4}(\Omega) ; L_{p}(\Omega)\right)\right) \quad \text { and } \quad \mathcal{F} \circ U_{n} \in C^{\nu}\left([0, T] ; L_{p}(\Omega)\right)
$$

with $\nu=\mu \frac{\gamma-\sigma}{2}$. Moreover, $U_{n}(0)=u\left(t_{n}\right) \in W_{p, B}^{4}(\Omega)$ and hence, by the linear theory, $U_{n} \in C^{1}\left([0, T] ; L_{p}(\Omega)\right) \cap C\left([0, T] ; W_{p, B}^{4}(\Omega)\right)$. Now define for $t_{n} \geq \bar{T}-\frac{T}{2}$

$$
\tilde{u}(t)= \begin{cases}u(t), & 0 \leq t<t_{n} \\ U_{n}\left(t-t_{n}\right), & t_{n} \leq t \leq t_{n}+T .\end{cases}
$$


As in the Proof of Theorem 4.4 one can now show that $\tilde{u}$ is a solution of $\dot{\tilde{u}}+\mathcal{A}(\tilde{u}) \tilde{u}=\mathcal{F}(\tilde{u})$ on $\left(0, t_{n}+T\right)$ that enjoys the regularity

$$
\tilde{u} \in C\left(\left[0, t_{n}+T\right] ; W_{p, B}^{4 \rho}(\Omega)\right) \cap C\left(\left(0, t_{n}+T\right] ; W_{p, B}^{4}(\Omega)\right) .
$$

Since $t_{n}+T \geq \bar{T}+\frac{T}{2}>\bar{T}$ this is a contradiction to the definition of $\bar{T}$.

Analogously one shows that for solutions in the sense of Theorem 5.4 the maximal time $\bar{T}$ of existence is characterised by $\bar{T}=\infty$ or

$$
\liminf _{t \nearrow \bar{T}} \frac{1}{\min _{\bar{\Omega}} u(t)}+\|u(t)\|_{h_{B}^{4 \gamma}(\bar{\Omega})}=\infty
$$

for all $\gamma \in(\sigma, 1]$.

\section{Acknowledgements}

Open Access funding provided by Projekt DEAL. The first author is grateful to the anonymous reviewer whose comments have improved the original version of the manuscript and to Joachim Escher and Lorenzo Giacomelli for fruitful discussions on the topic. Both authors have been supported by the Deutsche Forschungsgemeinschaft (DFG, German Research Foundation) through the collaborative research centre 'The mathematics of emerging effects' (CRC 1060, Projekt-ID 211504053) and the Hausdorff Center for Mathematics (GZ 2047/1, Projekt-ID 390685813).

Open Access. This article is licensed under a Creative Commons Attribution 4.0 International License, which permits use, sharing, adaptation, distribution and reproduction in any medium or format, as long as you give appropriate credit to the original author(s) and the source, provide a link to the Creative Commons licence, and indicate if changes were made. The images or other third party material in this article are included in the article's Creative Commons licence, unless indicated otherwise in a credit line to the material. If material is not included in the article's Creative Commons licence and your intended use is not permitted by statutory regulation or exceeds the permitted use, you will need to obtain permission directly from the copyright holder. To view a copy of this licence, visit http://creativecommons. org/licenses/by/4.0/.

Publisher's Note Springer Nature remains neutral with regard to jurisdictional claims in published maps and institutional affiliations.

\section{References}

[1] Acquistapace, P., Terreni, B.: Hölder classes with boundary condition as interpolation spaces. Math. Z. 195, 451-471 (1987)

[2] Acquistapace, P., Terreni, B.: On the abstract non-autonomous Cauchy problem in the case of constant domains. Ann. Mat. Pura Appl. 4(140), 1-55 (1985)

[3] Amann, H.: Linear and Quasilinear Parabolic Problems, Volume I: Abstract Linear Theory. Birkhäuser, Basel (1995) 
[4] Amann, H.: Nonhomogeneous linear and quasilinear elliptic and parabolic boundary value problems. In: Schmeisser, H.-J., Triebel, H. (eds.) Function Spaces, Differential Operators and Nonlinear Analysis. Teubner-Texte zur Math, pp. 9-126. Vieweg Teubner Verlag, Stuttgart (1993)

[5] Ansini, L., Giacomelli, L.: Shear-thinning liquid films: macroscopic and asymptotic behavior by quasi-self-similar solutions. Nonlinearity 256, 2147-2164 (2002)

[6] Ansini, L., Giacomelli, L.: Doubly nonlinear thin-film equations in one space dimension. Arch. Ration. Mech. Anal. 173, 89-131 (2004)

[7] Bird, R.B., Armstrong, R.C., Hassager, O.: Dynamics of Polymeric Liquids. Wiley, New York (1977)

[8] Bernis, F., Friedman, A.: Higher order nonlinear degenerate parabolic equations. J. Differ. Equ. 83, 179-206 (1990)

[9] Bertozzi, A.L., Pugh, M.: The lubrication approximation in thin viscous films: regularity and long time behavior of weak solutions. Commun. Pure Appl. Math. 49, 85-123 (1996)

[10] Beretta, E., Bertsch, M., Dal Passo, R.: Nonnegative solutions of a fourthorder nonlinear degenerate parabolic equation. Arch. Ration. Mech. Anal. Math. Model Numer. Anal. 129, 175-200 (1995)

[11] Chiricotto, M., Giacomelli, L.: Weak solutions to thin-film equations with contact-line friction. Interfaces Free Bound. 19, 243-271 (2017)

[12] DiBenedetto, E.: Degenerate Parabolic Equations. Springer, New York (1993)

[13] Degtyarev, S.: Classical solvability of the multidimensional free boundary problem for the thin-film equation with quadratic mobility in the case of partial wetting. Contin. Dyn. Syst. 37, 3625-3699 (2017)

[14] Eidel'man, S.D.: Parabolic Systems. North-Holland Publishing Company, Amsterdam (1969)

[15] Fischer, J.: Behaviour of free boundaries in thin-film flow: the regime of strong slippage and the regime of very weak slippage. Ann. Inst. H. Poincaré Anal. Non Linéaire 33, 1301-1327 (2016)

[16] Giacomelli, L., Gnann, M.V., Knüpfer, H., Otto, F.: Well-posedness for the Navier-slip thin-film equation in the case of complete wetting. J. Differ. Equ. 257, 15-81 (2014)

[17] Giacomelli, L., Gnann, M.V., Otto, F.: Regularity of source-type solutions to the thin-film equation with zero contact angle and mobility exponent between 3/2 and 3 European. J. Appl. Math. 24, 735-760 (2013)

[18] Giacomelli, L., Knüpfer, H.: A free boundary problem of fourth order: classical solutions in weighted Hölder spaces. Commun. Partial Differ. Equ. 35, 2059-2091 (2010) 
[19] Giacomelli, L., Knüpfer, H., Otto, F.: Smooth zero-contact-angle solutions to a thin-film equation around the steady state. J. Differ. Equ. 245, 1454-1506 (2008)

[20] Giacomelli, L., Otto, F.: Rigorous lubrication approximation. Interfaces Free Bound. 5, 483-592 (2003)

[21] Gilbarg, D., Trudinger, N.S.: Elliptic Partial Differential Equations of Second Order. Springer, Berlin (1998)

[22] Gnann, M.V.: Well-posedness and self-similar asymptotics for a thin-film equation. SIAM J. Math. Anal. 47, 2868-2902 (2015)

[23] Gnann, M.V.: On the regularity for the Navier-slip thin-film equation in the perfect wetting regime. Arch. Ration. Mech. Anal. 222, 1285-1337 (2016)

[24] Gnann, M.V., Petrache, M.: The Navier-slip thin-film equation for 3D fluid films: existence and uniqueness. J. Differ. Equ. 265, 5832-5958 (2018)

[25] King, J.R.: The spreading of power-law fluids. In: IUTAM Symposium on Free Surface Flows, pp. 153-160 (2001)

[26] King, J.R.: Two generalisations of the thin film equation. Math. Comput. Model. 34, 737-756 (2001)

[27] Knüpfer, H.: Well-posedness for the Navier-slip thin-film equation in the case of partial wetting. Arch. Ration. Mech. Anal. 218, 1083-1130 (2015)

[28] Knüpfer, H.: Well-posedness for a class of thin-film equations with general mobility in the regime of partial wetting. Commun. Pure Appl. Math. 64, 1263-1296 (2011)

[29] Knüpfer, H., Masmoudi, N.: Darcy's flow with prescribed contact angle: wellposedness and lubrication approximation. Arch. Ration. Mech. Anal. 218, 589$646(2015)$

[30] Ladyženskaja, O.A., Solonnikov, V.A., Ural'ceva, N.N.: Linear and quasilinear equations of parabolic type, Translation Mathematical Monographs, American Mathematical Society (1968)

[31] Lunardi, A.: Analytic Semigroups and Optimal Regularity in Parabolic Problems. Progress in Nonlinear Differential Equations and Their Applications, vol. 16. Birkhäuser, Basel (1995)

[32] Matsuhisa, S., Byron Bird, R.: Analytical and numerical solutions for laminar flow of the non-Newtonian ellis fluid. AIChE J. 11, 588-595 (1965)

[33] Málek, J., Rajagopal, K.R., Žabenský, J.: On power-law fluids with the powerlaw index proportional to the pressure. Appl. Mathe. Lett. 62, 118-123 (2016)

[34] Otto, F.: Lubrication approximation with prescribed non-zero contact angle. Commun. Partial Differ. Equ. 23, 2077-2164 (1998)

[35] Runst, T., Sickel, W.: Sobolev Spaces of Fractional Order, Nemitskiy Operators, and Partial Differential Equations. De Gruyter Series in Nonlinear Analysis and Applications, vol. 3. Walter de Gruyter \& Co., Berlin (1996) 
[36] Seis, C.: The thin-film equation close to self-similarity. Anal. PDE 11, 1303-1342 (2018)

[37] Simon, L.: Schauder estimates by scaling. Calc. Var. Partial Differ. Equ. 5, 391407 (1997)

[38] Sobolevskii, P.E.: Equations of parabolic type in Banach spaces. Am. Math. Soc. Trans. Ser. 2(49), 1-62 (1966)

[39] Solonnikov, V.A.: On boundary value problems for linear parabolic systems of differential equations of general form. Trudy Mat. Inst. Steklov. 83, 3-163 (1965). (Russian)

[40] Stewart, H.P.: Generation of analytic semigroups by strongly elliptic operators. Trans. Am. Math. Soc. 199, 141-162 (1974)

[41] Stewart, H.P.: Generation of analytic semigroups by strongly elliptic operators under general boundary conditions. Trans. Am. Math. Soc. 259, 299-310 (1980)

[42] Tanabe, H.: On the equation of evolution in a Banach space. Osaka Math. J. 12, 363-376 (1960)

[43] Weidner, D.E., Schwartz, L.W.: Contact-line motion of shear-thinning liquids. Phys. Fluids 6, 3535-3538 (1994)

Christina Lienstromberg and Stefan Müller

Institute of Applied Mathematics

University of Bonn

Endenicher Allee 60

53115 Bonn

Germany

e-mail: lienstromberg@iam.uni-bonn.de

Stefan Müller

e-mail: stefan.mueller@hcm.uni-bonn.de

Received: 13 November 2018.

Accepted: 4 February 2020. 